\title{
Simulations of Merging Helion Bunches on the AGS Injection Porch
}

\author{
C.J. Gardner
}

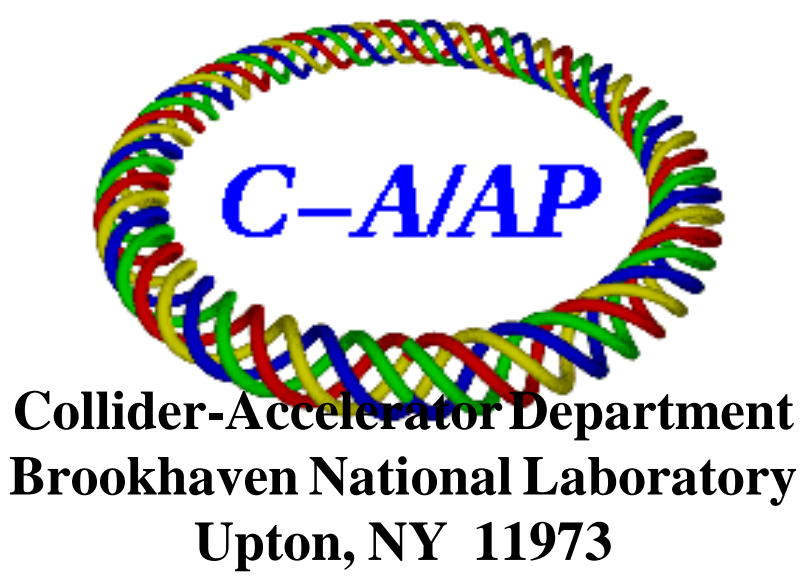

U.S. Department of Energy

Office of Science, Office of Nuclear Physics

Notice: This document has been authorized by employees of Brookhaven Science Associates, LLC under Contract No. DE-AC02-98CH10886 with the U.S. Department of Energy. The United States Government retains a nonexclusive, paid-up, irrevocable, world-wide license to publish or reproduce the published form of this document, or allow others to do so, for United States Government purposes. 


\title{
Simulations of Merging Helion Bunches on the AGS Injection Porch
}

\author{
C.J. Gardner
}

August 29, 2014

During the setup of helions for the FY2014 RHIC run it was discovered

that the standard scheme for merging bunches on the AGS injection porch required an injection kicker pulse shorter than what was available. To overcome this difficulty, K. Zeno [1] proposed and developed an interesting and unusual alternative which uses RF harmonic numbers 12, 4, 2 (rather than the standard $8,4,2$ ) to merge 8 helion bunches into 2 . In this note we carry out simulations that illustrate how the alternative scheme works and how it compares with the standard scheme. This is done in Sections 13 and 14. A scheme in which 6 bunches are merged into 1 is simulated in Section 15. This may be useful if more helions per merged bunch are needed in future runs.

General formulae for the simulations are given in Sections 9 through 12 .

For completeness, Sections 1 through 8 give a derivation of the turn-by-turn equations of longitudinal motion at constant magnetic field. The derivation is based on the work of MacLachlan [2]. The reader may wish to skip over these Sections and start with Section 9. 


\section{Synchronous Parameters}

Let $2 \pi R$ and $\rho$ be the circumference and radius-of-curvature of the design orbit in a given ring, and let $B$ and $2 \pi R_{s}$ be the magnetic field and orbit circumference for the synchronous particle. We assume that $B$ and $R_{s}$ are given and calculate the other synchronous particle parameters in terms of these. The radius-of-curvature of the synchronous particle is

$$
\rho_{s}=\rho\left(R_{s} / R\right)^{1 / \alpha}
$$

where

$$
\alpha=\frac{1}{\gamma_{t}^{2}}
$$

is the "momentum compaction" factor and $\gamma_{t}$ is the transition gamma of the ring. The momentum of the synchronous particle is given by

$$
c p_{s}=e Q B \rho_{s}
$$

where $e$ is the proton charge and $e Q$ is the charge of the particle. The energy is

$$
E_{s}=\sqrt{\left(c p_{s}\right)^{2}+m^{2} c^{4}}
$$

where $m$ is the mass of the particle. The synchronous $\beta, \gamma$ and angular velocity are

$$
\beta_{s}=c p_{s} / E_{s}, \quad \gamma_{s}=E_{s} /\left(m c^{2}\right), \quad \omega_{s}=c \beta_{s} / R_{s} .
$$

The revolution period and frequency of the synchronous particle are

$$
T_{s}=\frac{2 \pi}{\omega_{s}}, \quad f_{s}=\frac{1}{T_{s}} .
$$

We also define the phase slip factor

$$
\eta_{s}=\frac{1}{\gamma_{t}^{2}}-\frac{1}{\gamma_{s}^{2}}
$$




\section{Time Equation}

We consider a ring with a single RF gap. Let $T_{n}^{s}$ and $T_{n}$ be respectively the times at which the synchronous and asynchronous particles make their $n$th pass through the gap. The synchronous particle experiences no acceleration in the gap and revolves around the ring with constant angular velocity $\omega_{s}$. Thus

$$
T_{n+1}^{s}=T_{n}^{s}+\frac{2 \pi}{\omega_{s}}
$$

and taking $T_{1}^{s}=0$ it follows that

$$
T_{n+1}^{s}=n T_{s} .
$$

Similarly, for the asynchronous particle we have

$$
T_{n+1}=T_{n}+\frac{2 \pi}{\omega_{n}}
$$

where $\omega_{n}$ is the angular velocity just after the $n$th pass through the gap.

Defining

$$
t_{n+1}=T_{n+1}-T_{n+1}^{s}, \quad t_{n}=T_{n}-T_{n}^{s}
$$

we then have

$$
t_{n+1}=t_{n}+2 \pi\left(\frac{1}{\omega_{n}}-\frac{1}{\omega_{s}}\right)=t_{n}+\left(\frac{\omega_{s}-\omega_{n}}{\omega_{n}}\right) T_{s} .
$$

\section{$3 \quad$ Energy Equation}

Now let $E_{n}$ be the energy of the asynchronous particle just after its $n$th pass through the gap. Then we have

$$
E_{n+1}=E_{n}+e Q V\left(T_{n+1}\right),
$$

where $V(T)$ is the voltage across the gap at time $T$. Since the synchronous particle undergoes no acceleration we must have

$$
V\left(T_{n}^{s}\right)=0
$$

for all $n$. We assume that

$$
V\left(T+T_{s} / h\right)=V(T)
$$


where $h$ is a positive integer called the fundamental harmonic. It then follows that

$$
V\left(T+n T_{s}\right)=V(T) .
$$

In terms of $E_{n}$, the other asynchronous parameters are

$$
c p_{n}=\sqrt{E_{n}^{2}-m^{2} c^{4}}, \quad \beta_{n}=c p_{n} / E_{n}
$$

and

$$
\rho_{n}=\frac{c p_{n}}{e Q B}, \quad R_{n}=R\left(\rho_{n} / \rho\right)^{\alpha}, \quad \omega_{n}=c \beta_{n} / R_{n} .
$$

Define

$$
e_{n+1}=E_{n+1}-E_{s}, \quad e_{n}=E_{n}-E_{s} .
$$

Then using

$$
T_{n+1}=t_{n+1}+T_{n+1}^{s}=t_{n+1}+n T_{s}
$$

we have

$$
V\left(T_{n+1}\right)=V\left(t_{n+1}\right)
$$

and

$$
e_{n+1}=e_{n}+e Q V\left(t_{n+1}\right) \text {. }
$$

This, together with

$$
t_{n+1}=t_{n}+\left(\frac{\omega_{s}-\omega_{n}}{\omega_{n}}\right) T_{s}
$$

gives the turn-by-turn longitudinal motion of the asynchronous particle.

\section{Symplectic Map}

The Jacobian matrix elements for the map from $\left(t_{n}, e_{n}\right)$ to $\left(t_{n+1}, e_{n+1}\right)$ are

$$
\begin{gathered}
\frac{\partial t_{n+1}}{\partial t_{n}}=1, \quad \frac{\partial t_{n+1}}{\partial e_{n}}=2 \pi \frac{\partial\left(1 / \omega_{n}\right)}{\partial e_{n}} \\
\frac{\partial e_{n+1}}{\partial t_{n}}=e Q V^{\prime}, \quad \frac{\partial e_{n+1}}{\partial e_{n}}=1+2 \pi e Q V^{\prime} \frac{\partial\left(1 / \omega_{n}\right)}{\partial e_{n}}
\end{gathered}
$$

where $V^{\prime}$ is the derivative of $V$ with respect to $T$ at time $T_{n+1}$. Thus we have

$$
\left(\frac{\partial t_{n+1}}{\partial t_{n}}\right)\left(\frac{\partial e_{n+1}}{\partial e_{n}}\right)-\left(\frac{\partial t_{n+1}}{\partial e_{n}}\right)\left(\frac{\partial e_{n+1}}{\partial t_{n}}\right)=1
$$

and the map is symplectic. This guarantees that the map preserves area. 


\section{Approximate Time Equation}

Writing

$$
\frac{\omega_{s}}{\omega_{n}}=\left\{1+\left(\frac{\omega_{n}-\omega_{s}}{\omega_{s}}\right)\right\}^{-1}
$$

and expanding we have

$$
\frac{\omega_{s}}{\omega_{n}}=1-\left(\frac{\omega_{n}-\omega_{s}}{\omega_{s}}\right)+\left(\frac{\omega_{n}-\omega_{s}}{\omega_{s}}\right)^{2}-\left(\frac{\omega_{n}-\omega_{s}}{\omega_{s}}\right)^{3}+\cdots .
$$

To first order in $p_{n}-p_{s}$ and $E_{n}-E_{s}$ we also have

$$
\left(\frac{\omega_{n}-\omega_{s}}{\omega_{s}}\right)=-\eta_{s}\left(\frac{p_{n}-p_{s}}{p_{s}}\right)=-\eta_{s}\left(\frac{E_{n}-E_{s}}{\beta_{s}^{2} E_{s}}\right) .
$$

Thus to first order

$$
\frac{\omega_{s}}{\omega_{n}}=1-\left(\frac{\omega_{n}-\omega_{s}}{\omega_{s}}\right)=1+\left(\frac{\eta_{s}}{\beta_{s}^{2} E_{s}}\right) e_{n}
$$

and (23) becomes

$$
t_{n+1}=t_{n}+T_{s}\left(\frac{\eta_{s}}{\beta_{s}^{2} E_{s}}\right) e_{n}
$$

This together with

$$
e_{n+1}=e_{n}+e Q V\left(t_{n+1}\right)
$$

again produces a symplectic map from $\left(t_{n}, e_{n}\right)$ to $\left(t_{n+1}, e_{n+1}\right)$.

\section{$6 \quad$ Phase and $W$ Equations}

Let us now introduce new variables

$$
\phi_{n}=h \omega_{s} t_{n}, \quad W_{n}=\frac{e_{n}}{h \omega_{s}} .
$$

Here $\phi_{n}$ is the phase that corresponds to time $t_{n}$, and $W_{n}$ is defined so that the transformation from $\left(t_{n}, e_{n}\right)$ to $\left(\phi_{n}, W_{n}\right)$ is symplectic. Note that $\phi_{n}$ varies from $-\pi$ to $+\pi$ as $t_{n}$ varies from $-T_{s} /(2 h)$ to $+T_{s} /(2 h)$. In terms of the new variables equations (31) and (32) become

$$
\phi_{n+1}=\phi_{n}+T_{s}\left(\frac{h^{2} \omega_{s}^{2} \eta_{s}}{\beta_{s}^{2} E_{s}}\right) W_{n}
$$


and

$$
W_{n+1}=W_{n}+T_{s}\left(\frac{e Q}{2 \pi h}\right) V\left(\frac{\phi_{n+1}}{h \omega_{s}}\right) .
$$

Defining

$$
a=\left(\frac{h^{2} \omega_{s}^{2} \eta_{s}}{\beta_{s}^{2} E_{s}}\right)
$$

and

$$
F\left(\phi_{n+1}\right)=\left(\frac{e Q}{2 \pi h}\right) V\left(\frac{\phi_{n+1}}{h \omega_{s}}\right)
$$

we then have

$$
\phi_{n+1}=\phi_{n}+a T_{s} W_{n}
$$

and

$$
W_{n+1}=W_{n}+T_{s} F\left(\phi_{n+1}\right) .
$$

These equations again generate a sympletic map. Note that since $V(0)=0$ and $V\left(T+T_{s} / h\right)=V\left(T_{s}\right)$ we have

$$
F(0)=0, \quad F(\phi+2 \pi)=F(\phi) .
$$

Since we are interested in the momentum deviation $p_{n}-p_{s}$, it is useful to have an expression for this in terms of $W_{n}$. Using (29) and (33) we find

$$
\left(\frac{p_{n}-p_{s}}{p_{s}}\right)=\left(\frac{E_{n}-E_{s}}{\beta_{s}^{2} E_{s}}\right)=\left(\frac{h \omega_{s}}{\beta_{s}^{2} E_{s}}\right) W_{n}
$$

\section{$7 \quad$ Fixed Points of the Motion}

Fixed points of the motion are points $\left(\phi_{f}, W_{f}\right)$ that are unchanged by (38) and (39). For these points we must have

$$
F\left(\phi_{f}\right)=0, \quad W_{f}=0 .
$$

Since $F(0)=0$ we see that the point $(0,0)$ is a fixed point.

If a particle launched near a fixed point stays close to the fixed point, the fixed point is said to be stable. In the Appendix we show that below transition $(a<0)$ a fixed point is stable if

$$
F^{\prime}\left(\phi_{f}\right)>0
$$

and unstable if

$$
F^{\prime}\left(\phi_{f}\right)<0
$$


where the primes denote differentiation with respect to $\phi$. Similarly above transition $(a>0)$ a fixed point will be stable if

$$
F^{\prime}\left(\phi_{f}\right)<0
$$

and unstable if

$$
F^{\prime}\left(\phi_{f}\right)>0
$$

\section{RF Buckets}

Consider the hamiltonian

$$
H(\phi, W)=\frac{1}{2} a W^{2}+U(\phi)
$$

where

$$
\frac{\partial U}{\partial \phi}=-F(\phi)
$$

The equations of motion are

$$
\begin{gathered}
\frac{d \phi}{d t}=\frac{\partial H}{\partial W}=a W \\
\frac{d W}{d t}=-\frac{\partial H}{\partial \phi}=F(\phi)
\end{gathered}
$$

and we have

$$
\frac{d H}{d t}=\frac{\partial H}{\partial t}=\frac{\partial U}{\partial t} .
$$

The hamiltonian is therefore a constant of the motion if $U$ has no explicit dependence on time. This constraint can be used to determine the extent of the regions of bounded motion generated by $H$.

The fixed points are the same as those for the motion generated by (38) and (39). They are given by (42). Let

$$
U_{\phi \phi}=U^{\prime \prime}\left(\phi_{f}\right)
$$

where the primes denote differentiation with respect to $\phi$. Then below transition $(a<0)$ the fixed point will be stable if $U_{\phi \phi}<0$ and unstable if $U_{\phi \phi}>0$. Similarly above transition $(a>0)$ the fixed point will be stable if $U_{\phi \phi}>0$ and unstable if $U_{\phi \phi}<0$. 
Let $H_{u}$ be the value of $H$ at an unstable fixed point $\left(\phi_{u}, 0\right)$. Then

$$
H_{u}=U\left(\phi_{u}\right)
$$

and the equation

$$
H(\phi, W)=H_{u}
$$

can be solved to obtain

$$
W^{2}(\phi)=\frac{2}{a}\left\{U\left(\phi_{u}\right)-U(\phi)\right\} .
$$

The curve $W(\phi)$ is called the separatrix. Differentiating $W^{2}(\phi)$ with respect to $\phi$ we obtain

$$
\frac{d W^{2}}{d \phi}=-\frac{2}{a} U^{\prime}(\phi)
$$

and

$$
\frac{d^{2} W^{2}}{d^{2} \phi}=-\frac{2}{a} U^{\prime \prime}(\phi)
$$

which shows that $W^{2}(\phi)$ reaches a local maximum at each stable fixed point. The area around a stable fixed point and bounded by the separatrix is an RF bucket. This is the extent of the region of bounded motion around the fixed point. The beam contained in the RF bucket is called a bunch. The height $W_{b}$ of the bucket is given by

$$
W_{b}^{2}=\frac{2}{a}\left\{U\left(\phi_{u}\right)-U\left(\phi_{s}\right)\right\}
$$

where $\phi_{s}$ is the stable fixed point phase.

First-order symplectic integration [3] of (49) and (50) from time $t$ to time $t+T_{s}$ yields

$$
\phi\left(t+T_{s}\right)=\phi(t)+a T_{s} W(t)
$$

and

$$
W\left(t+T_{s}\right)=W(t)+T_{s} F\left(\phi\left(t+T_{s}\right)\right) .
$$

These are the same as (38) and (39). This suggests that the motion generated by the hamiltonian approximates that generated by (38) and (39). To the extent that this is true, the regions of bounded motion generated by $H$ will approximate those generated by (38) and (39). 


\section{Capturing Unbunched Beam into RF Buckets}

For simulations that start with bunched beam we want an initial beam distribution that consists of $\hat{h}$ bunches in $\hat{h}$ adjacent harmonic $\hat{h} h$ buckets. Here $\hat{h} h$ is the total number of RF buckets around the ring and $\hat{h}$ is the number of buckets in $1 / h$ of the ring. To obtain the desired $\hat{h}$ bunches we start with a uniform distribution of unbunched beam consisting of an 80-by-80 array of particles which cover the region

$$
-\phi_{I} \leq \phi \leq \phi_{I}, \quad-W_{I} \leq W \leq W_{I}
$$

where

$$
\phi_{I}=\pi
$$

and

$$
W_{I}=\frac{E_{I}}{h \omega_{s}}=\frac{E_{I} T_{s}}{2 \pi h} .
$$

Here $E_{I}$ is the energy half-width of the unbunched beam. As defined by (33), the phase $\phi$ varies from $-\pi$ to $+\pi$ as the time deviation $t$ varies from $-T_{s} /(2 h)$ to $+T_{s} /(2 h)$. The region defined by (61) therefore occupies $1 / h$ of the ring as desired. The area of the region is

$$
\epsilon=\left(2 \phi_{I}\right)\left(2 W_{I}\right)=2 E_{I} T_{s} / h .
$$

This is the longitudinal emittance of the unbunched beam (in $1 / h$ of the ring).

To capture the beam into $\hat{h}$ adjacent harmonic $\hat{h} h$ buckets we take

$$
F(\phi)=-A \sin \hat{h} \phi
$$

in (38) and (39). During capture $A$ is raised slowly and quadratically from zero to $A_{C}$ over time interval $0 \leq T \leq T_{C}$ according to

$$
A(T)=A_{C}\left(\frac{T}{T_{C}}\right)^{2}
$$

where

$$
A_{C}=\frac{e Q V_{C}}{2 \pi h}
$$

and $V_{C}$ is the RF harmonic $\hat{h} h$ voltage at time $T_{C}$. The time $T_{C}$ is chosen to be long enough to ensure that the longitudinal emittance of the $\hat{h}$ bunches is as close to that of the initial distribution of unbunched beam as practical. 


\section{Formulae for the Standard 4 to 1 Merge}

Here we divide the $\hat{h} h$ buckets into $h$ groups of $\hat{h}=4$ adjacent buckets and consider the merge of the 4 bunches in a given group into 1 bunch. If all of the $\hat{h} h$ buckets are occupied then the gap of time available for the injection kicker pulse is

$$
T_{G}=2\left(\frac{T_{s}}{\hat{h} h}\right)-T_{W}
$$

where $T_{W}$ is the bunch width and $T_{s}$ is the revolution period.

For the merge we take

$$
F(\phi)=A_{1} \sin \phi-A_{2} \sin 2 \phi-A_{4} \sin 4 \phi
$$

in (38) and (39). The parameters $A_{1}, A_{2}$ and $A_{4}$ are either zero or positive. They are varied slowly during the merging process. Note again that the phase $\phi$ varies from $-\pi$ to $+\pi$ as the time deviation $t$ varies from $-T_{s} /(2 h)$ to $+T_{s} /(2 h)$.

In order to satisfy (48) we take

$$
U(\phi)=A_{1} \cos \phi-\frac{1}{2} A_{2} \cos 2 \phi-\frac{1}{4} A_{4} \cos 4 \phi
$$

which gives

$$
U^{\prime \prime}(\phi)=-A_{1} \cos \phi+2 A_{2} \cos 2 \phi+4 A_{4} \cos 4 \phi .
$$

This is positive at fixed point phases

$$
\phi_{u}= \pm \pi
$$

which shows that these are unstable fixed point phases below transition.

We then have

$$
U\left(\phi_{u}\right)=-A_{1}-\frac{A_{2}}{2}-\frac{A_{4}}{4}
$$

which can be used in (55) to obtain the separatrix.

Starting with 4 bunches in 4 adjacent harmonic $4 h$ buckets, the merge is done in two steps. First the 4 bunches are merged into 2 and then the 2 are merged into 1 . The merge of 4 bunches into 2 takes place over the time interval $0 \leq T \leq T_{K}$. During this time $A_{1}$ is held at zero, $A_{2}$ is raised linearly from zero to $A_{K}$, and $A_{4}$ is lowered linearly from $A_{C}$ to zero. Thus

$$
A_{1}(T)=0
$$


and

$$
A_{2}(T)=A_{K}\left(\frac{T}{T_{K}}\right), \quad A_{4}(T)=A_{C}\left(\frac{T_{K}-T}{T_{K}}\right) .
$$

Here

$$
A_{K}=\frac{e Q V_{K}}{2 \pi h}, \quad A_{C}=\frac{e Q V_{C}}{2 \pi h}
$$

where $V_{K}$ is the harmonic $2 h$ voltage at time $T_{K}$ and $V_{C}$ is the harmonic $4 h$ voltage at time 0 .

The merge of 2 bunches into 1 takes place over the time interval

$T_{K} \leq T \leq T_{L}$. During this time $A_{4}$ is held at zero, $A_{1}$ is raised linearly from zero to $A_{L}$, and $A_{2}$ is lowered linearly from $A_{K}$ to zero. Thus

$$
A_{1}(T)=A_{L}\left(\frac{T-T_{K}}{T_{L}-T_{K}}\right), \quad A_{2}(T)=A_{K}\left(\frac{T_{L}-T}{T_{L}-T_{K}}\right)
$$

and

$$
A_{4}(T)=0
$$

Here

$$
A_{L}=\frac{e Q V_{L}}{2 \pi h}
$$

where $V_{L}$ is the harmonic $h$ voltage at time $T_{L}$.

\section{Formulae for the Alternative 4 to 1 Merge}

In this case we divide the $\hat{h} h$ buckets into $h$ groups of $\hat{h}=6$ adjacent buckets and consider the merge of the 4 inner bunches of a given group into 1 bunch. The motivation here is that if the 2 outer buckets in each group are unoccupied, they provide time for the injection kicker pulse. The total gap of time available is

$$
T_{G}=4\left(\frac{T_{s}}{\hat{h} h}\right)-T_{W}
$$

where $T_{W}$ is the bunch width and $T_{s}$ is the revolution period. For harmonic $\hat{h} h=12$ this turns out to be just long enough for the kicker pulse [4].

For this merge we take

$$
F(\phi)=A_{1} \sin \phi-A_{2} \sin 2 \phi-A_{6} \sin 6 \phi
$$

in (38) and (39). The parameters $A_{1}, A_{2}$, and $A_{6}$ are either zero or positive. They are varied slowly during the merging process. Note again 
that the phase $\phi$ varies from $-\pi$ to $+\pi$ as the time deviation $t$ varies from $-T_{s} /(2 h)$ to $+T_{s} /(2 h)$.

In order to satisfy (48) we take

$$
U(\phi)=A_{1} \cos \phi-\frac{1}{2} A_{2} \cos 2 \phi-\frac{1}{6} A_{6} \cos 6 \phi
$$

which gives

$$
U^{\prime \prime}(\phi)=-A_{1} \cos \phi+2 A_{2} \cos 2 \phi+6 A_{6} \cos 6 \phi .
$$

This is positive at fixed point phases

$$
\phi_{u}= \pm \pi
$$

which shows that these are unstable fixed point phases below transition.

We then have

$$
U\left(\phi_{u}\right)=-A_{1}-\frac{A_{2}}{2}-\frac{A_{6}}{6}
$$

which can be used in (55) to obtain the separatrix.

At fixed point phase

$$
\phi_{f}=0
$$

we have

$$
U^{\prime \prime}\left(\phi_{f}\right)=-A_{1}+2 A_{2}+6 A_{6} .
$$

This gives an unstable fixed point (below transition) if

$$
A_{1}<2 A_{2}+6 A_{6} .
$$

In this case one has unstable fixed point phase

$$
\phi_{u}=0
$$

and

$$
U\left(\phi_{u}\right)=A_{1}-\frac{A_{2}}{2}-\frac{A_{6}}{6}
$$

which can be used in (55) to obtain the associated separatrix.

For $0 \leq T \leq T_{K}$ we take

$$
A_{1}(T)=\mathcal{F} A_{L}\left(\frac{T}{T_{K}}\right), \quad A_{2}(T)=A_{K}\left(\frac{T}{T_{K}}\right)
$$

and

$$
A_{6}(T)=A_{C}\left(\frac{T_{K}-T}{T_{K}}\right)
$$


where

$$
0 \leq \mathcal{F} \leq 1
$$

The parameter $\mathcal{F}$ is adjusted to keep the 4 inner bunches separated from the phase space of the 2 outer buckets, and to minimize the gross emittance of the final merged bunch.

For $T_{K} \leq T \leq T_{L}$ we take

$$
A_{6}(T)=0, \quad A_{2}(T)=A_{K}\left(\frac{T_{L}-T}{T_{L}-T_{K}}\right)
$$

and

$$
A_{1}(T)=\mathcal{F} A_{L}+(1-\mathcal{F}) A_{L}\left(\frac{T-T_{K}}{T_{L}-T_{K}}\right)
$$

Here

$$
A_{L}=\frac{e Q V_{L}}{2 \pi h}, \quad A_{K}=\frac{e Q V_{K}}{2 \pi h}, \quad A_{C}=\frac{e Q V_{C}}{2 \pi h}
$$

where $V_{L}, V_{K}$, and $V_{C}$ are the harmonic $h, 2 h$, and $6 h$ voltages at times $T_{L}, T_{K}$, and 0 , respectively.

\section{Formulae for a 6 to 1 Merge}

In this case we divide the $\hat{h} h$ buckets into $h$ groups of $\hat{h}=6$ adjacent buckets and consider the merge of the 6 bunches in a given group into 1

bunch. The motivation here is to get more helions per merged bunch. If all of the $\hat{h} h$ buckets are occupied then the gap of time available for the injection kicker pulse is

$$
T_{G}=2\left(\frac{T_{s}}{\hat{h} h}\right)-T_{W}
$$

where $T_{W}$ is the bunch width and $T_{s}$ is the revolution period.

For this merge we take

$$
F(\phi)=A_{1} \sin \phi-A_{2} \sin 2 \phi+A_{3} \sin 3 \phi-A_{6} \sin 6 \phi
$$

in (38) and (39). The parameters $A_{1}, A_{2}, A_{3}$, and $A_{6}$ are either zero or positive. They are varied slowly during the merging process. Note again that the phase $\phi$ varies from $-\pi$ to $+\pi$ as the time deviation $t$ varies from $-T_{s} /(2 h)$ to $+T_{s} /(2 h)$. 
In order to satisfy (48) we take

$$
U(\phi)=A_{1} \cos \phi-\frac{1}{2} A_{2} \cos 2 \phi+\frac{1}{3} A_{3} \cos 3 \phi-\frac{1}{6} A_{6} \cos 6 \phi
$$

which gives

$$
U^{\prime \prime}(\phi)=-A_{1} \cos \phi+2 A_{2} \cos 2 \phi-3 A_{3} \cos 3 \phi+6 A_{6} \cos 6 \phi .
$$

This is positive at fixed point phases

$$
\phi_{u}= \pm \pi
$$

which shows that these are unstable fixed point phases below transition. We then have

$$
U\left(\phi_{u}\right)=-A_{1}-\frac{A_{2}}{2}-\frac{A_{3}}{3}-\frac{A_{6}}{6}
$$

which can be used in (55) to obtain the separatrix.

For the case $A_{6}=0$ we have

$$
\begin{gathered}
F(\phi)=A_{1} \sin \phi-A_{2} \sin 2 \phi+A_{3} \sin 3 \phi \\
U(\phi)=A_{1} \cos \phi-\frac{1}{2} A_{2} \cos 2 \phi+\frac{1}{3} A_{3} \cos 3 \phi
\end{gathered}
$$

and

$$
U^{\prime \prime}(\phi)=-A_{1} \cos \phi+2 A_{2} \cos 2 \phi-3 A_{3} \cos 3 \phi .
$$

Using

$$
\sin 2 \phi=2 \cos \phi \sin \phi
$$

and

$$
\sin 3 \phi=3 \sin \phi-4 \sin ^{3} \phi
$$

we then have

$$
F(\phi)=\left\{A_{1}-2 A_{2} \cos \phi+A_{3}\left(4 \cos ^{2} \phi-1\right)\right\} \sin \phi
$$

which shows that in addition to the unstable fixed point phases (101) we have fixed point phases given by

$$
A_{1}-2 A_{2} \cos \phi_{f}+A_{3}\left(4 \cos ^{2} \phi_{f}-1\right)=0 .
$$

Solving this for $\cos \phi_{f}$ gives

$$
\cos \phi_{f}=\frac{A_{2}}{4 A_{3}} \pm \frac{1}{4 A_{3}} \sqrt{A_{2}^{2}+4 A_{3}\left(A_{3}-A_{1}\right)} .
$$


To evaluate $U(\phi)$ and $U^{\prime \prime}(\phi)$ at $\phi_{f}$ we use

$$
\cos 2 \phi=2 \cos ^{2} \phi-1
$$

and

$$
\cos 3 \phi=4 \cos ^{3} \phi-3 \cos \phi
$$

which give

$$
U(\phi)=A_{1} \cos \phi-\frac{A_{2}}{2}\left(2 \cos ^{2} \phi-1\right)+\frac{A_{3}}{3}\left(4 \cos ^{3} \phi-3 \cos \phi\right)
$$

and

$$
U^{\prime \prime}(\phi)=-A_{1} \cos \phi+2 A_{2}\left(2 \cos ^{2} \phi-1\right)-3 A_{3}\left(4 \cos ^{3} \phi-3 \cos \phi\right) .
$$

For the values of $A_{1}, A_{2}$, and $A_{3}$ considered here, $U^{\prime \prime}\left(\phi_{f}\right)$ is positive for the phase given by the upper sign in (110). This is then the phase for an unstable fixed point below transition. The lower sign gives the phase for a stable fixed point below transition. Thus using

$$
\cos \phi_{u}=\frac{A_{2}}{4 A_{3}}+\frac{1}{4 A_{3}} \sqrt{A_{2}^{2}+4 A_{3}\left(A_{3}-A_{1}\right)}
$$

in (113) gives $U\left(\phi_{u}\right)$ which can be used in (55) to obtain the associated separatrix.

Starting with 6 bunches in 6 adjacent harmonic $6 h$ buckets, the merge is done in three steps. First the 6 bunches are merged into 3 . This is a straightforward 2 to 1 merge. Next the 3 bunches are moved toward one another but are kept in separate buckets. Finally, as soon as the bunches touch one another, they are allowed to mix and merge into a single bunch. The 3 to 1 merge is based on the the work of Garoby [5,6]. A similar scheme was developed by M. Blaskiewicz and J.M. Brennan for merging 24 bunches of gold ions into 4 bunches on the AGS injection porch [7].

The merge of 6 bunches into 3 takes place over the time interval $0 \leq T \leq T_{K}$. During this time $A_{1}$ and $A_{2}$ are held at zero, $A_{3}$ is raised linearly from zero to $A_{M}$, and $A_{6}$ is lowered linearly from $A_{C}$ to zero. Thus we have

$$
A_{1}(T)=0, \quad A_{2}(T)=0
$$

and

$$
A_{3}(T)=A_{M}\left(\frac{T}{T_{K}}\right), \quad A_{6}(T)=A_{C}\left(\frac{T_{K}-T}{T_{K}}\right) .
$$


Here

$$
A_{M}=\frac{e Q V_{M}}{2 \pi h}, \quad A_{C}=\frac{e Q V_{C}}{2 \pi h}
$$

where $V_{M}$ is the harmonic $3 h$ voltage at time $T=T_{K}$ and $V_{C}$ is the harmonic $6 h$ voltage at time $T=0$.

The first part of the merge of 3 bunches into 1 takes place over the time interval $T_{K} \leq T \leq T_{L 1}$. During this time $A_{6}$ is held at zero, $A_{3}$ is lowered linearly from $A_{M}$ to $A_{M 1}$, and $A_{1}$ is raised linearly from zero to $A_{L 1}$. The parameter $A_{2}$ is raised linearly from zero to $A_{K 1}$. Thus we have

$$
\begin{gathered}
A_{6}(T)=0 \\
A_{3}(T)=A_{M}\left(\frac{T_{L 1}-T}{T_{L 1}-T_{K}}\right)+A_{M 1}\left(\frac{T-T_{K}}{T_{L 1}-T_{K}}\right)
\end{gathered}
$$

and

$$
A_{1}(T)=A_{L 1}\left(\frac{T-T_{K}}{T_{L 1}-T_{K}}\right), \quad A_{2}(T)=A_{K 1}\left(\frac{T-T_{K}}{T_{L 1}-T_{K}}\right)
$$

where

$$
A_{K 1}=\frac{e Q V_{K 1}}{2 \pi h}, \quad A_{L 1}=\frac{e Q V_{L 1}}{2 \pi h}, \quad A_{M 1}=\frac{e Q V_{M 1}}{2 \pi h} .
$$

Here $V_{K 1}, V_{L 1}$, and $V_{M 1}$ are the harmonic $2 h, h$, and $3 h$ voltages, respectively, at time $T_{L 1}$.

The second part of the 3 to 1 merge takes place over the time interval $T_{L 1} \leq T \leq T_{L 2}$. During this time $A_{6}$ continues to be held at zero, $A_{3}$ is lowered linearly from $A_{M 1}$ to $A_{M 2}$, and $A_{2}$ is lowered linearly from $A_{K 1}$ to $A_{K 2}$. The parameter $A_{1}$ is held at $A_{L 1}$. Thus

$$
\begin{aligned}
& A_{3}(T)=A_{M 1}\left(\frac{T_{L 2}-T}{T_{L 2}-T_{L 1}}\right)+A_{M 2}\left(\frac{T-T_{L 1}}{T_{L 2}-T_{L 1}}\right) \\
& A_{2}(T)=A_{K 1}\left(\frac{T_{L 2}-T}{T_{L 2}-T_{L 1}}\right)+A_{K 2}\left(\frac{T-T_{L 1}}{T_{L 2}-T_{L 1}}\right)
\end{aligned}
$$

and

$$
A_{1}(T)=A_{L 1}, \quad A_{6}(T)=0
$$

where

$$
A_{K 2}=\frac{e Q V_{K 2}}{2 \pi h}, \quad A_{M 2}=\frac{e Q V_{M 2}}{2 \pi h} .
$$


Here $V_{K 2}$ and $V_{M 2}$ are the harmonic $2 h$ and $3 h$ voltages, respectively, at time $T_{L 2}$.

The final part of the 3 to 1 merge takes place over the time interval $T_{L 2} \leq T \leq T_{F}$. During this time $A_{6}$ continues to be held at zero, $A_{3}$ is lowered linearly from $A_{M 2}$ to zero, $A_{2}$ is lowered linearly from $A_{K 2}$ to zero, and $A_{1}$ continues to be held at $A_{L 1}$. Thus

$$
A_{3}(T)=A_{M 2}\left(\frac{T_{F}-T}{T_{F}-T_{L 2}}\right), \quad A_{2}(T)=A_{K 2}\left(\frac{T_{F}-T}{T_{F}-T_{L 2}}\right)
$$

and

$$
A_{1}(T)=A_{L 1}, \quad A_{6}(T)=0 .
$$

\section{Simulation of Standard 4 to 1 Merge}

In this scheme we take harmonic $\hat{h} h=8$ on the AGS injection porch and populate all 8 buckets with helion bunches from Booster. This gives $h=2$ groups of $\hat{h}=4$ adjacent bunches. The 4 bunches in each group are merged into 2 bunches by bringing on harmonic $4=2 h$ voltage with $R F$ cavity KL while reducing the harmonic $8=4 h$ voltage to zero. The 2 bunches are then merged into 1 by bringing on harmonic $2=1 h$ voltage with RF cavity L10 while reducing the harmonic $4=2 h$ voltage to zero.

For the simulation we start with 4 adjacent bunches of helions. These are obtained from a uniform distribution of unbunched beam as described in Section 9 . We use capture time $T_{C}=400 \mathrm{~ms}$ and harmonic 8 voltage $V_{C}=40 \mathrm{kV}$. The harmonic 8 frequency is $8 f_{s}=2.51 \mathrm{MHz}$. The longitudinal emittance of the unbunched beam is taken to be $0.80 \mathrm{eV}$ s per nucleon. Figure 1 shows the resulting bunches in 4 buckets on the porch. One can see by inspection that the emittance of the 4 bunches is close to that of the initial distribution. This gives an emittance of $0.2 \mathrm{eV} \mathrm{s}$ per nucleon per bunch. Note also that taking bunch width $T_{W}=90 \mathrm{~ns}$ in (68) gives time gap

$$
T_{G}=707 \mathrm{~ns}
$$

which is too short (by some $300 \mathrm{~ns}$ ) for the present injection kicker pulse. This means that in practice only 7 of the 8 buckets can be filled with bunches from Booster.

Figure 2 shows the RF voltages for the 4 to 1 merge. These are given by the formulae of Section 10 with $h$ set equal to 2 . Here the 4 to 2 merge 
takes place over the time interval $0 \leq T \leq 80 \mathrm{~ms}$. The 2 to 1 merge takes place over the interval $80 \leq T \leq 160 \mathrm{~ms}$.

Figure 3 shows the "potential" $U\left(\phi_{u}\right)-U(\phi)$ at various times during the merge. Here $U\left(\phi_{u}\right)$ and $U(\phi)$ are defined in Sections 8 and 10.

The merge from 4 to 2 bunches is shown in Figures 4 and $\mathbf{5}$. The merge from 2 to 1 bunches is shown in Figures 6 and 7 . One can see by inspection that the emittance of the final merged bunch is close to that (0.80 eV s per nucleon) of the initial distribution of unbunched beam. The bunch is easily held in the harmonic 2 bucket with voltage $V_{L}=20 \mathrm{kV}$.

\section{Simulation of Alternative 4 to 1 Merge}

In this scheme we take harmonic $\hat{h} h=12$ on the AGS injection porch. This gives $h=2$ groups of $\hat{h}=6$ adjacent buckets. The 4 inner buckets of each group are populated with helion bunches from Booster. The 2 outer buckets of each group are left empty to provide time for the injection kicker pulse. The 4 bunches in each group are merged into 2 bunches by bringing on harmonic $4=2 h$ and $2=1 h$ voltages with RF cavities KL and L10, respectively, while reducing the harmonic $12=6 h$ voltage to zero. The 2 bunches are then merged into 1 by reducing the harmonic $4=2 h$ voltage to zero.

For the simulation we start with 6 adjacent bunches of helions. These are obtained from a uniform distribution of unbunched beam as described in Section 9 . We use capture time $T_{C}=200 \mathrm{~ms}$ and harmonic 12 voltage $V_{C}=47 \mathrm{kV}$. The harmonic 12 frequency is $12 f_{s}=3.765 \mathrm{MHz}$. The longitudinal emittance of the unbunched beam is taken to be $1.2 \mathrm{eV}$ s per nucleon. Figure 8 shows the resulting bunches in 6 buckets on the porch. One can see by inspection that the emittance of the 6 bunches is close to that of the initial distribution. This gives an emittance of $0.2 \mathrm{eV} \mathrm{s}$ per nucleon per bunch. Note also that taking bunch width $T_{W}=90 \mathrm{~ns}$ in (80) gives time gap

$$
T_{G}=972 \mathrm{~ns}
$$

which is just long enough for the present injection kicker pulse [4].

Figure 9 shows the RF voltages for the 4 to 1 merge. These are given by the formulae of Section 11 with $h$ set equal to 2 . Here the parameter $\mathcal{F}$ (defined in Section 11) has been set equal to 0.95. This setting keeps the inner 4 bunches separated from the outer 2 during the merging process and 
minimizes the gross emittance of the final merged bunch.

Figure 10 shows the "potential" $U\left(\phi_{u}\right)-U(\phi)$ at various times during the merge. Here $U\left(\phi_{u}\right)$ and $U(\phi)$ are defined in Sections 8 and 11.

The merge from 4 to 2 bunches is shown in Figures $\mathbf{1 1}$ and 12. Here we see that the brown separatrix keeps the inner 4 bunches separated from the outer 2. The two outer bunches (which are not present in practice) form a halo around the inner 4 .

The merge from 2 to 1 bunches is shown in Figures 13 and 14. One can see by inspection that the emittance of the final merged bunch is close to that $(0.80 \mathrm{eVs}$ per nucleon) of the inner 4 bunches of the initial

distribution of bunched beam. The bunch is easily held in the harmonic 2 bucket with voltage $V_{L}=22 \mathrm{kV}$.

Comparing Figures $\mathbf{7}$ and $\mathbf{1 4}$ one sees that alternative 4 to 1 merge produces a merged bunch of the same size and quality as the standard 4 to 1 merge.

\section{Simulation of 6 to 1 Merge}

In this scheme we again take harmonic $\hat{h} h=12$ on the AGS injection porch. This gives $h=2$ groups of $\hat{h}=6$ adjacent buckets. The 6 buckets of each group are populated with helion bunches from Booster. The 6 bunches in each group are merged into 3 bunches by bringing on harmonic $6=3 h$ while reducing the harmonic $12=6 h$ voltage to zero. The 3 bunches are then merged into 1 by manipulating harmonics $6=3 h$, $4=2 h$, and $2=1 h$ as described in Section 12. Harmonics 4 and 2 are provided by the KL and L10 cavities respectively.

For the simulation we start with 6 adjacent bunches of helions. These are obtained from a uniform distribution of unbunched beam as described in Section 9 . We use capture time $T_{C}=200 \mathrm{~ms}$ and harmonic 12 voltage $V_{C}=47 \mathrm{kV}$. The harmonic 12 frequency is $12 f_{s}=3.765 \mathrm{MHz}$. The longitudinal emittance of the unbunched beam is taken to be $1.2 \mathrm{eV}$ s per nucleon. Figure 15 shows the resulting bunches in 6 buckets on the porch. One can see by inspection that the emittance of the 6 bunches is close to that of the initial distribution. This gives an emittance of $0.2 \mathrm{eV} \mathrm{s}$ per nucleon per bunch. Note also that taking bunch width $T_{W}=90 \mathrm{~ns}$ in (97) gives time gap

$$
T_{G}=441 \mathrm{~ns} .
$$


This is too short for the present injection kicker pulse, but still allows for 6 of the 12 buckets to be filled.

Figures 16 and 17 show the RF voltages for the 6 to 1 merge. These are given by the formulae of Section 12 with $h$ set equal to 2 . Here the 6 to 3 merge takes place over the time interval $0 \leq T \leq 30 \mathrm{~ms}$. The 3 to 1 merge takes place over the interval $30 \leq T \leq 120 \mathrm{~ms}$.

Figures 18 and 19 show the "potential" $U\left(\phi_{u}\right)-U(\phi)$ at various times during the merge. Here $U\left(\phi_{u}\right)$ and $U(\phi)$ are defined in Sections 8 and 12 .

The merge from 6 to 3 bunches is shown in Figures 20 and 21.

Figures 22 and $\mathbf{2 3}$ show the 3 bunches one sixth and one third of the way through the 3 to 1 merge. In both figures the bunches are kept separated by the brown separatrix. In Figure 23 the bunches are nearly touching but are still in separate buckets.

In Figure 24 the bunches are halfway through the 3 to 1 merge. Here the bunches have started to mix with one another.

In Figure 25 the bunches are two thirds of the way through the 3 to 1 merge. The bunches continue to mix and merge.

The completion of the 3 to 1 merge is shown in Figure 26. The magnified view in Figure 27 shows a tightly merged bunch. This is the final result of the 6 to 1 merge.

Note that if the 2 outer buckets in Figure $\mathbf{1 5}$ are left empty, then the 6 to 1 merge becomes another alternative scheme for merging 4 bunches into 1 . In this case, however, the phase space of the 2 outer buckets mixes with the 4 inner bunches and dilutes the gross emittance. One ends up with the bunch shown in Figure 28. (This was obtained by simply turning off the black and brown points in Figure 26.) The gross emittance of the bunch is $6 / 4$ times larger than that obtained with the alternative 4 to 1 scheme of Section 14. 


\section{Appendix}

For motion near a fixed point $\left(\phi_{f}, 0\right)$ we have

$$
F(\phi)=-k\left(\phi-\phi_{f}\right)
$$

where

$$
k=-F^{\prime}\left(\phi_{f}\right)
$$

Equations (38) and (39) then become

$$
\phi_{n+1}-\phi_{f}=\left(\phi_{n}-\phi_{f}\right)+a T_{s} W_{n}
$$

and

$$
W_{n+1}=W_{n}-k T_{s}\left(\phi_{n}-\phi_{f}\right)-a k T_{s}^{2} W_{n} .
$$

Defining

$$
\Phi=\phi-\phi_{f}
$$

and

$$
\mathbf{X}_{n+1}=\left(\begin{array}{c}
\Phi_{n+1} \\
W_{n+1}
\end{array}\right), \quad \mathbf{X}_{n}=\left(\begin{array}{c}
\Phi_{n} \\
W_{n}
\end{array}\right)
$$

we then have

$$
\mathbf{X}_{n+1}=\mathbf{M X}_{n}
$$

where

$$
\mathbf{M}=\left(\begin{array}{cc}
1 & a T_{s} \\
-k T_{s} & 1-a k T_{s}^{2}
\end{array}\right) .
$$

The matrix $\mathbf{M}$ has unit determinant and its trace is

$$
\mathcal{T}=2-a k T_{s}^{2} .
$$

It can be expressed in Courant-Snyder [8] form

$$
\mathbf{M}=\left(\begin{array}{cc}
C+\mathcal{A} S & \mathcal{B} S \\
-\mathcal{G S} & C-\mathcal{A} S
\end{array}\right)
$$

where

$$
\begin{gathered}
C=1-\frac{1}{2} a k T_{s}^{2}, \quad S^{2}=1-C^{2} \\
\mathcal{B} S=a T_{s}, \quad 2 \mathcal{A} S=a k T_{s}^{2}, \quad \mathcal{G} S=k T_{s}
\end{gathered}
$$

and

$$
\mathcal{B G}-\mathcal{A}^{2}=1
$$




\subsection{Conserved Quadratic Form}

Defining

$$
\mathbf{E}=\left(\begin{array}{rr}
\mathcal{B} & -\mathcal{A} \\
-\mathcal{A} & \mathcal{G}
\end{array}\right), \quad \mathbf{E}^{-1}=\left(\begin{array}{cc}
\mathcal{G} & \mathcal{A} \\
\mathcal{A} & \mathcal{B}
\end{array}\right)
$$

and

$$
\mathbf{T}=\mathbf{M}^{T} \mathbf{E}^{-1} \mathbf{M}
$$

where the superscript $T$ denotes the transpose of the matrix, we have

$$
\begin{gathered}
\mathbf{T}=\left(\begin{array}{cc}
C+\mathcal{A} S & -\mathcal{G} S \\
\mathcal{B} S & C-\mathcal{A} S
\end{array}\right)\left(\begin{array}{cc}
\mathcal{G} & \mathcal{A} \\
\mathcal{A} & \mathcal{B}
\end{array}\right)\left(\begin{array}{cc}
C+\mathcal{A} S & \mathcal{B} S \\
-\mathcal{G} S & C-\mathcal{A} S
\end{array}\right) \\
\mathbf{T}=\left(\begin{array}{cc}
C+\mathcal{A} S & -\mathcal{G} S \\
\mathcal{B} S & C-\mathcal{A} S
\end{array}\right)\left(\begin{array}{cc}
\mathcal{G} C & S+\mathcal{A} C \\
\mathcal{A} C-S & \mathcal{B} C
\end{array}\right)
\end{gathered}
$$

and

$$
\mathbf{T}=\left(\begin{array}{cc}
\mathcal{G} & \mathcal{A} \\
\mathcal{A} & \mathcal{B}
\end{array}\right)
$$

Thus

$$
\mathbf{M}^{T} \mathbf{E}^{-1} \mathbf{M}=\mathbf{E}^{-1}
$$

and

$$
\mathbf{X}_{n+1}^{T} \mathbf{E}^{-1} \mathbf{X}_{n+1}=\mathbf{X}_{n}^{T} \mathbf{M}^{T} \mathbf{E}^{-1} \mathbf{M} \mathbf{X}_{n}=\mathbf{X}_{n}^{T} \mathbf{E}^{-1} \mathbf{X}_{n}
$$

Here we have quadratic forms

$$
\mathbf{X}_{n+1}^{T} \mathbf{E}^{-1} \mathbf{X}_{n+1}=\mathcal{G} \Phi_{n+1}^{2}+2 \mathcal{A} \Phi_{n+1} W_{n+1}+\mathcal{B} W_{n+1}^{2}
$$

and

$$
\mathbf{X}_{n}^{T} \mathbf{E}^{-1} \mathbf{X}_{n}=\mathcal{G} \Phi_{n}^{2}+2 \mathcal{A} \Phi_{n} W_{n}+\mathcal{B} W_{n}^{2}
$$

and we can write

$$
G\left(\Phi_{n+1}, W_{n+1}\right)=G\left(\Phi_{n}, W_{n}\right)
$$

where

$$
G(\Phi, W)=\mathcal{G} \Phi^{2}+2 \mathcal{A} \Phi W+\mathcal{B} W^{2}
$$

This shows that $G(\Phi, W)$ is a conserved quantity for the turn-by-turn motion generated by (134) and (135). 


\subsection{Stable Fixed Point}

We assume that $k<0$ below transition and $k>0$ above transition. Then since $a<0$ below transition and $a>0$ above transition, we have

$$
a k>0 \text {. }
$$

We assume further that $|k|$ is small enough to ensure that

$$
|\mathcal{T}|<2
$$

It then follows that $|C|<1$ and $S^{2}>0$. The parameters $S, \mathcal{A}, \mathcal{B}$, and $\mathcal{G}$ are then all real numbers. The sign of $S$ is chosen so that $\mathcal{B}>0$. We then have

$$
G(\Phi, W)=\mathcal{E}
$$

where $\mathcal{E}$ is a real number. With the help of (144) we have

$$
\Phi^{2}+(\mathcal{A} \Phi+\mathcal{B} W)^{2}=\mathcal{E B}
$$

and

$$
W^{2}+(\mathcal{G} \Phi+\mathcal{A} W)^{2}=\mathcal{E} \mathcal{G} .
$$

The maximum possible $\Phi$ is then

$$
\Phi_{M}=\sqrt{\mathcal{E B}}
$$

and the corresponding $W$ is

$$
W\left(\Phi_{M}\right)=-\frac{\mathcal{A}}{\mathcal{B}} \Phi_{M}
$$

Similarly the maximum possible $W$ is

$$
W_{M}=\sqrt{\mathcal{E} \mathcal{G}}
$$

and the corresponding $\Phi$ is

$$
\Phi\left(W_{M}\right)=-\frac{\mathcal{A}}{\mathcal{G}} W_{M}
$$

Here

$$
-\frac{\mathcal{A}}{\mathcal{B}}=-\frac{1}{2} k T_{s}, \quad-\frac{\mathcal{A}}{\mathcal{G}}=-\frac{1}{2} a T_{s} .
$$

This analysis shows that under the conditions assumed, a particle launched close to the fixed point will stay close to the fixed point. The fixed point is then said to be stable. 
Note that (158) defines an ellipse with area $\pi \mathcal{E}$ and Courant-Snyder parameters $\mathcal{A}, \mathcal{B}$ and $\mathcal{G}$. Since we have assumed that $k<0$ below transition we also have $\mathcal{A}<0$. The ellipse is therefore rotated clockwise with respect to the $\Phi$ and $W$ axes.

Note also that defining

$$
\mathcal{H}(\Phi, W)=\frac{S}{2 T_{s}} G(\Phi, W)
$$

and using the identities

$$
\frac{S \mathcal{B}}{2 T_{s}}=\frac{a}{2}, \quad \frac{2 \mathcal{A} S}{2 T_{s}}=\frac{1}{2} a k T_{s}, \quad \frac{S \mathcal{G}}{2 T_{s}}=\frac{k}{2}
$$

we have

$$
\mathcal{H}(\Phi, W)=\frac{1}{2} a W^{2}+\frac{1}{2} k \Phi^{2}+\frac{1}{2} a k T_{s} \Phi W .
$$

This is a conserved quantity for the motion generated by (134) and (135). It shows that the hamiltonian

$$
H=\frac{1}{2} a W^{2}+\frac{1}{2} k \Phi^{2}
$$

is not conserved. In fact we have

$$
H(\Phi, W)=\mathcal{H}(\Phi, W)-\frac{1}{2} a k T_{s} \Phi W
$$

which we can write as

$$
H(\Phi, W)=\frac{S \mathcal{E}}{2 T_{s}}-\frac{1}{2} a k T_{s} \Phi W
$$

where

$$
\mathcal{E}=G(\Phi, W) .
$$

The first term on the right hand side of (171) is constant but the second term varies with $\Phi$ and $W$.

\subsection{Explicit Turn-by-Turn Motion}

Now let

$$
\psi=\arcsin S
$$

where $S$ is given by (142). Then

$$
C=\cos \psi, \quad S=\sin \psi
$$


and following Courant and Snyder [8] we can write

$$
\mathbf{M}=\mathbf{I} \cos \psi+\mathbf{J} \sin \psi
$$

where

$$
\mathbf{I}=\left(\begin{array}{ll}
1 & 0 \\
0 & 1
\end{array}\right), \quad \mathbf{J}=\left(\begin{array}{rr}
\mathcal{A} & \mathcal{B} \\
-\mathcal{G} & -\mathcal{A}
\end{array}\right)
$$

Here

$$
\mathbf{J}^{2}=-\mathbf{I}
$$

and it follows that

$$
\{\mathbf{I} \cos \psi+\mathbf{J} \sin \psi\}\{\mathbf{I} \cos \chi+\mathbf{J} \sin \chi\}=\mathbf{I} \cos (\psi+\chi)+\mathbf{J} \sin (\psi+\chi) .
$$

Thus we have

$$
\mathbf{M}^{n}=\mathbf{I} \cos n \psi+\mathbf{J} \sin n \psi
$$

and

$$
\begin{gathered}
\Phi_{n}=\left(C_{n}+\mathcal{A} S_{n}\right) \Phi_{0}+\mathcal{B} S_{n} W_{0} \\
W_{n}=-\mathcal{G} S_{n} \Phi_{0}+\left(C_{n}-\mathcal{A} S_{n}\right) W_{0}
\end{gathered}
$$

where

$$
C_{n}=\cos n \psi, \quad S_{n}=\sin n \psi
$$

and $\Phi_{0}$ and $W_{0}$ are initial values of $\phi$ and $W$. Collecting terms we have

$$
\begin{gathered}
\Phi_{n}=\Phi_{0} C_{n}+\left(\mathcal{A} \Phi_{0}+\mathcal{B} W_{0}\right) S_{n} \\
W_{n}=W_{0} C_{n}-\left(\mathcal{G} \Phi_{0}+\mathcal{A} W_{0}\right) S_{n}
\end{gathered}
$$

and therefore

$$
\begin{aligned}
\Phi_{n} & =\sqrt{\mathcal{E B}}\left\{C_{n} \cos \xi-S_{n} \sin \xi\right\}=\sqrt{\mathcal{E B}} \cos (n \psi+\xi) \\
W_{n} & =\sqrt{\mathcal{E} \mathcal{G}}\left\{C_{n} \sin \chi+S_{n} \cos \chi\right\}=\sqrt{\mathcal{E G}} \sin (n \psi+\chi)
\end{aligned}
$$

where

$$
\begin{aligned}
& \mathcal{E B}=\Phi_{0}^{2}+\left(\mathcal{A} \Phi_{0}+\mathcal{B} W_{0}\right)^{2} \\
& \mathcal{E} \mathcal{G}=W_{0}^{2}+\left(\mathcal{G} \Phi_{0}+\mathcal{A} W_{0}\right)^{2}
\end{aligned}
$$

and

$$
\begin{aligned}
\sqrt{\mathcal{E B}} \cos \xi=\Phi_{0}, & \sqrt{\mathcal{E B}} \sin \xi=-\left(\mathcal{A} \Phi_{0}+\mathcal{B} W_{0}\right) \\
\sqrt{\mathcal{E} \mathcal{G}} \sin \chi=W_{0}, \quad \sqrt{\mathcal{E} \mathcal{G}} \cos \chi & =-\left(\mathcal{G} \Phi_{0}+\mathcal{A} W_{0}\right) .
\end{aligned}
$$

These equations give explicitly the turn-by-turn motion near the stable fixed point. 


\subsection{Synchrotron Oscillation Tune and Period}

The synchrotron tune is defined to be

$$
Q_{s}=\frac{|\psi|}{2 \pi} .
$$

The synchrotron oscillation period is then

$$
P_{s}=\frac{T_{s}}{Q_{s}} .
$$

For sufficiently small $a k T_{s}^{2}$ we have

$$
\psi=-T_{s} \sqrt{a k}
$$

which gives

$$
Q_{s}=\frac{T_{s}}{2 \pi} \sqrt{a k}, \quad P_{s}=\frac{2 \pi}{\sqrt{a k}} .
$$

For the case

$$
F(\phi)=\left(\frac{e Q V_{G}}{2 \pi h}\right) \sin \phi
$$

we have stable fixed point phase $\phi_{s}=0$ (below transition) and

$$
a k=-\frac{e Q V_{G}}{2 \pi h}\left(\frac{h^{2} \omega_{s}^{2} \eta_{s}}{\beta_{s}^{2} E_{s}}\right)=-\frac{e Q V_{G}}{2 \pi h}\left(\frac{h^{2} c^{2} \eta_{s}}{R_{s}^{2} E_{s}}\right) .
$$

\subsection{Unstable Fixed Point}

For all of the above work we have assumed that $k<0$ below transition and $k>0$ above transition. If instead we assume that $k>0$ below transition and $k<0$ above transition then

$$
a k<0
$$

and we see from (140) that the trace of $\mathbf{M}$ is greater than 2 . One then finds that a particle launched close to the fixed point will not stay close to the fixed point. The fixed point is then said to be unstable.

We again have conserved quadratic form (159) which we can write as

$$
\Phi^{2}-(i \mathcal{A} \Phi+i \mathcal{B} W)^{2}=\mathcal{E} \mathcal{B} .
$$


But now $\mathcal{A}, \mathcal{B}$, and $\mathcal{E}$ are pure imaginary numbers which means that $i \mathcal{A}$, $i \mathcal{B}$, and $\mathcal{E B}$ are all real. Equation (198) therefore defines a hyperbola. The asymptotes are given by

$$
i \mathcal{A} \Phi+i \mathcal{B} W= \pm \Phi
$$

which we can write as

$$
W=\frac{-i \mathcal{A} \pm 1}{i \mathcal{B}} \Phi .
$$

For a particle on either of the asymptotes we have $\mathcal{E}=0$. Since $\mathcal{E}$ is a conserved quantity, the particle stays on the asymptote.

Again following Courant and Snyder [8] we can write

$$
\mathbf{M}=C \mathbf{I}+S \mathbf{J}
$$

where

$$
\mathbf{I}=\left(\begin{array}{ll}
1 & 0 \\
0 & 1
\end{array}\right), \quad \mathbf{J}=\left(\begin{array}{rr}
\mathcal{A} & \mathcal{B} \\
-\mathcal{G} & -\mathcal{A}
\end{array}\right)
$$

and

$$
\mathbf{J}^{2}=-\mathbf{I}
$$

Here $C$ and $S$ are given by (142) and since $C>1$, we can find real $\psi$ such that

$$
C=\cosh \psi, \quad S=i \sinh \psi
$$

We then have

$$
\mathbf{M}=\mathbf{I} \cosh \psi+i \mathbf{J} \sinh \psi
$$

and

$$
\begin{array}{r}
\{\mathbf{I} \cosh \psi+i \mathbf{J} \sinh \psi\}\{\mathbf{I} \cosh \chi+i \mathbf{J} \sinh \chi\} \\
=\mathbf{I} \cosh (\psi+\chi)+i \mathbf{J} \sinh (\psi+\chi) .
\end{array}
$$

It then follows that

$$
\mathbf{M}^{n}=\mathbf{I} \cosh n \psi+i \mathbf{J} \sinh n \psi
$$

and

$$
\mathbf{M}^{n}=\left(\begin{array}{cc}
\cosh n \psi+i \mathcal{A} \sinh n \psi & i \mathcal{B} \sinh n \psi \\
-i \mathcal{G} \sinh n \psi & \cosh n \psi-i \mathcal{A} \sinh n \psi
\end{array}\right)
$$

This gives the turn-by-turn motion near the unstable fixed point. 


\section{References}

[1] K.L. Zeno, Booster-AGS-He3-2014 elog, 22-23 May 2014.

[2] J.A. MacLachlan, "Difference Equations for Longitudinal Motion in a Synchrotron", Fermilab internal report FNAL FN-529, December 15, 1989; "Differential Equations for Longitudinal Motion in a Synchrotron", Fermilab internal report FNAL FN-532, January 25, 1990.

[3] H. Yoshida, Numerical Integration Methods, Handbook of Accelerator Physics and Engineering, Edited by A.W. Chao and M. Tigner, World Scientific, 1999, pp. 85-87.

[4] K.L. Zeno, Booster-AGS-He3-2014 elog, 23 May 2014, entry 15:02.

[5] R. Garoby, "Bunch Merging and Splitting Techniques in the Injectors for High Energy Hadron Colliders", CERN/PS 98-048(RF), 1 October 1998.

[6] R. Garoby, S. Hancock, and J.L. Vallet, "Demonstration of Bunch Triple Splitting in the CERN PS", Proceedings of EPAC 2000, pp. 304-306.

[7] C.J. Gardner, et al., "Setup and Performance of the RHIC Injector Accelerators for the 2007 Run with Gold Ions", Proceedings of PAC07, pp.1862-1864.

[8] E.D. Courant and H.S. Snyder, Annals of Physics 3, 1-48 (1958). 


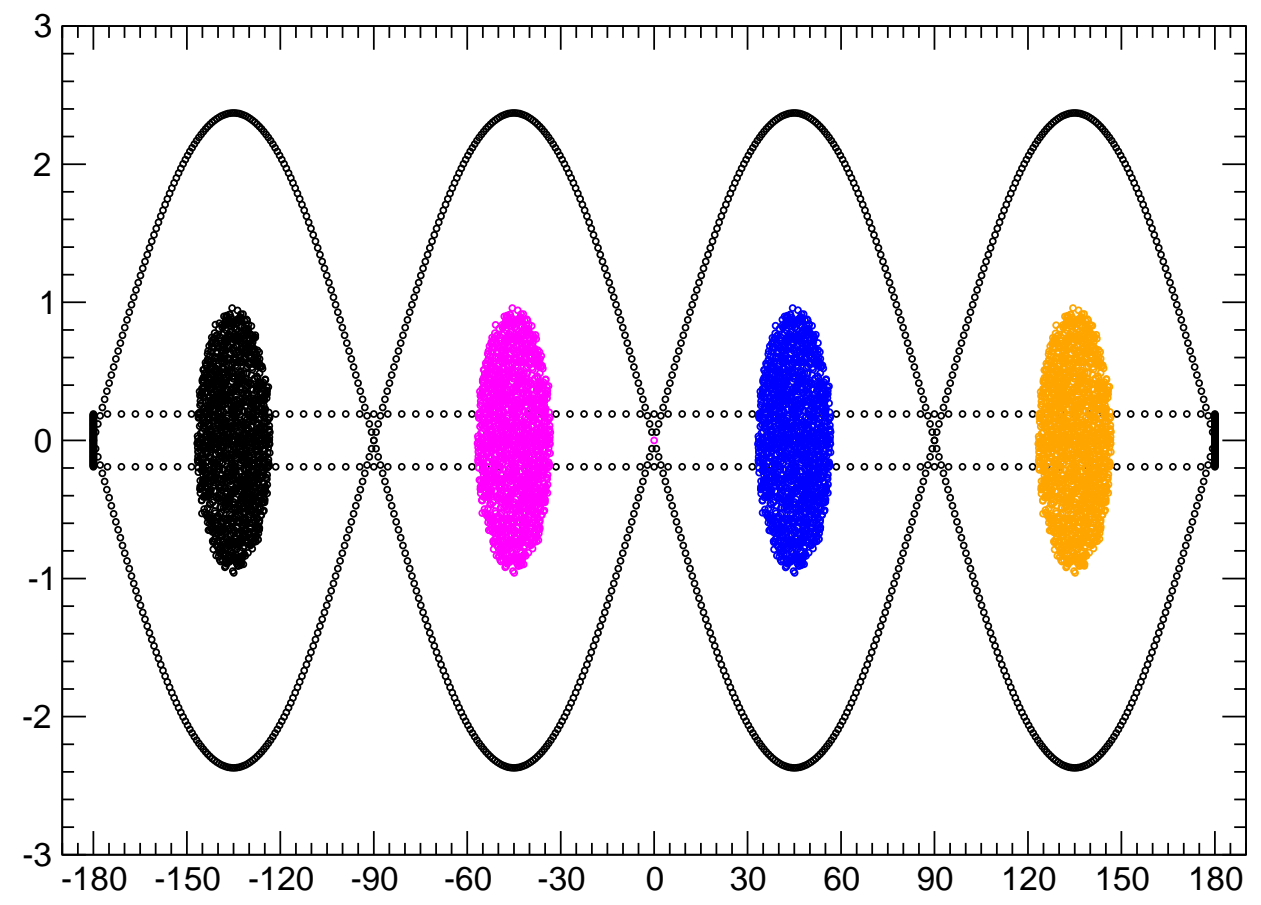

Figure 1: Four bunches of helions in harmonic 8 buckets on the AGS injection porch. The harmonic 8 frequency is $8 f_{s}=2.51 \mathrm{MHz}$. The RF voltage is $V_{C}=40 \mathrm{kV}$. The black rectangle is the border of the uniform distribution used to make the bunches. The longitudinal emittance of the distribution is $0.80 \mathrm{eV} \mathrm{s}$ per nucleon. One can see by inspection that the emittance of the 4 bunches is close to that of the initial distribution. This gives an emittance of $0.2 \mathrm{eVs}$ per nucleon per bunch. The horizontal axis is the phase $\phi$ in degrees. The vertical axis is $W$ in units of eV s. 


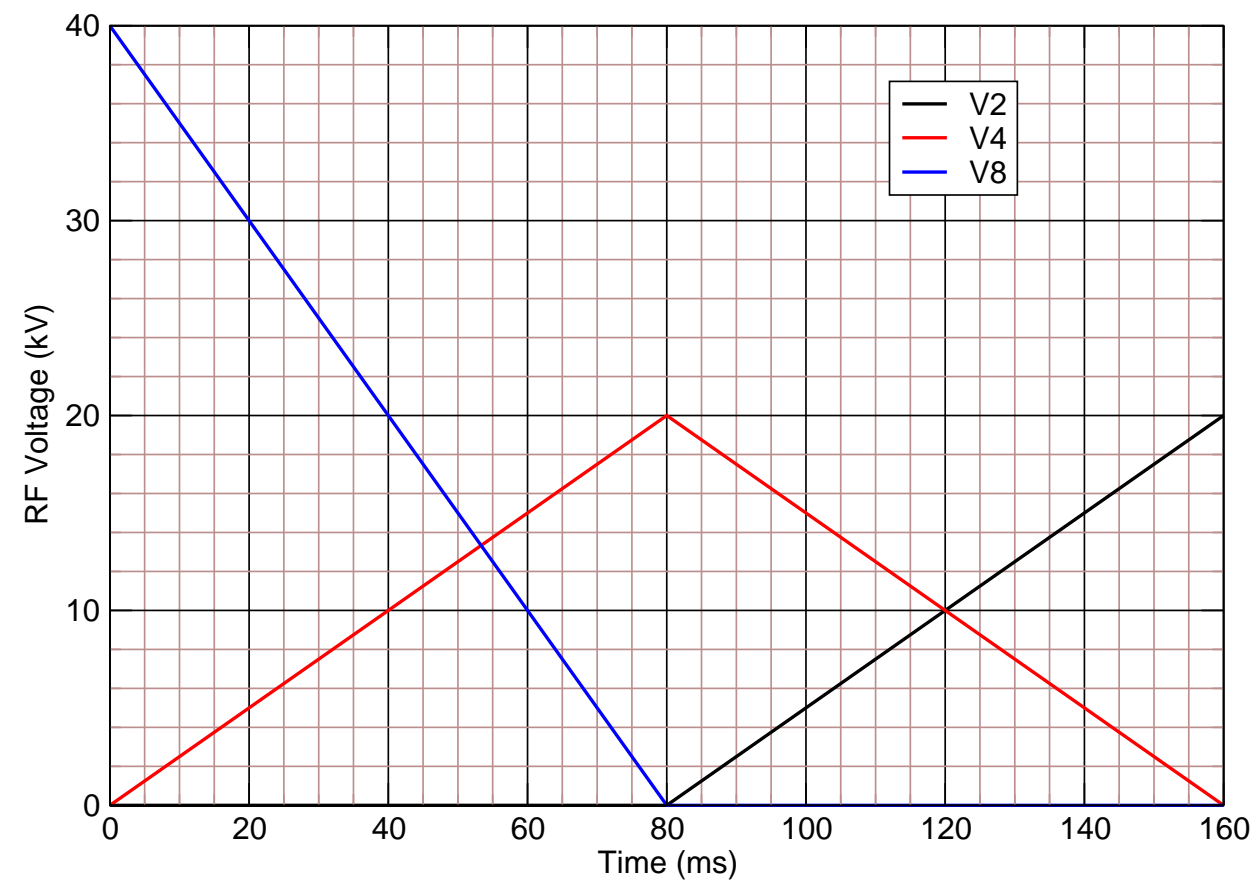

Figure 2: RF voltages for the standard 4 to 1 merge. Here the black, red, and blue curves are the harmonic $2=1 h, 4=2 h$, and $8=4 h$ voltages respectively. These are given by the formulae of Section 10 with $h$ set equal to 2 . Here the harmonic 8,4 , and 2 voltages are $V_{C}=40 \mathrm{kV}, V_{K}=20$ $\mathrm{kV}$, and $V_{L}=20 \mathrm{kV}$ respectively. The times $T_{K}$ and $T_{L}$ are 80 and $160 \mathrm{~ms}$ respectively. 


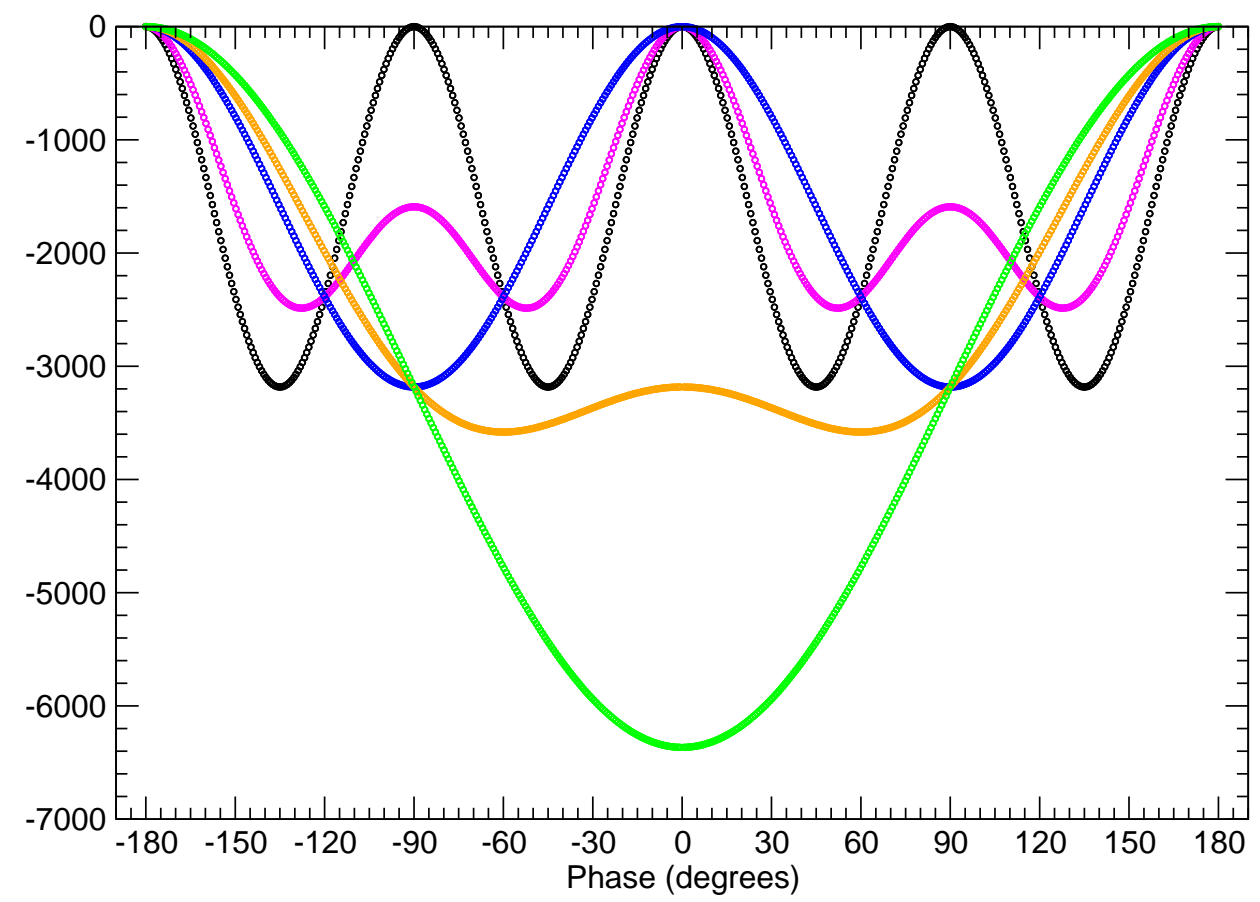

Figure 3: Plots of the "potential" $U\left(\phi_{u}\right)-U(\phi)$ at various times during the standard 4 to 1 merge. Here $U\left(\phi_{u}\right)$ and $U(\phi)$ are defined in Sections 8 and 10. The black, magenta, blue, orange, and green curves are $U\left(\phi_{u}\right)-U(\phi)$ at times $0,40,80,120$, and $160 \mathrm{~ms}$ respectively. The local minima of the curves correspond to stable fixed points. The local maxima correspond to unstable fixed points. 


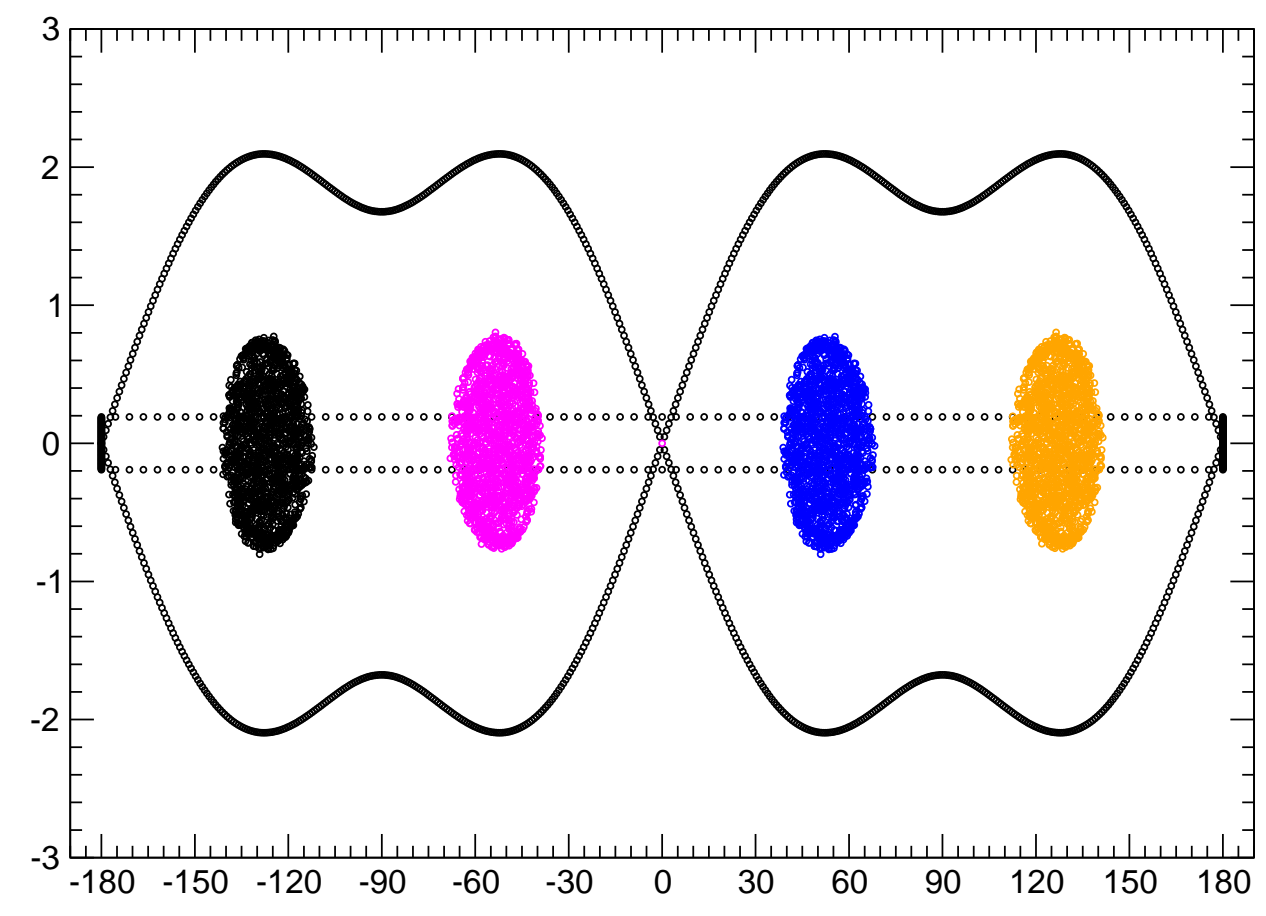

Figure 4: Halfway through the 4 to 2 merge of the bunches in Figure 1. This is at time $40 \mathrm{~ms}$ in Figure $\mathbf{2}$ and corresponds to the magenta curve in Figure 3. The horizontal axis is the phase $\phi$ in degrees. The vertical axis is $W$ in units of eVs. 


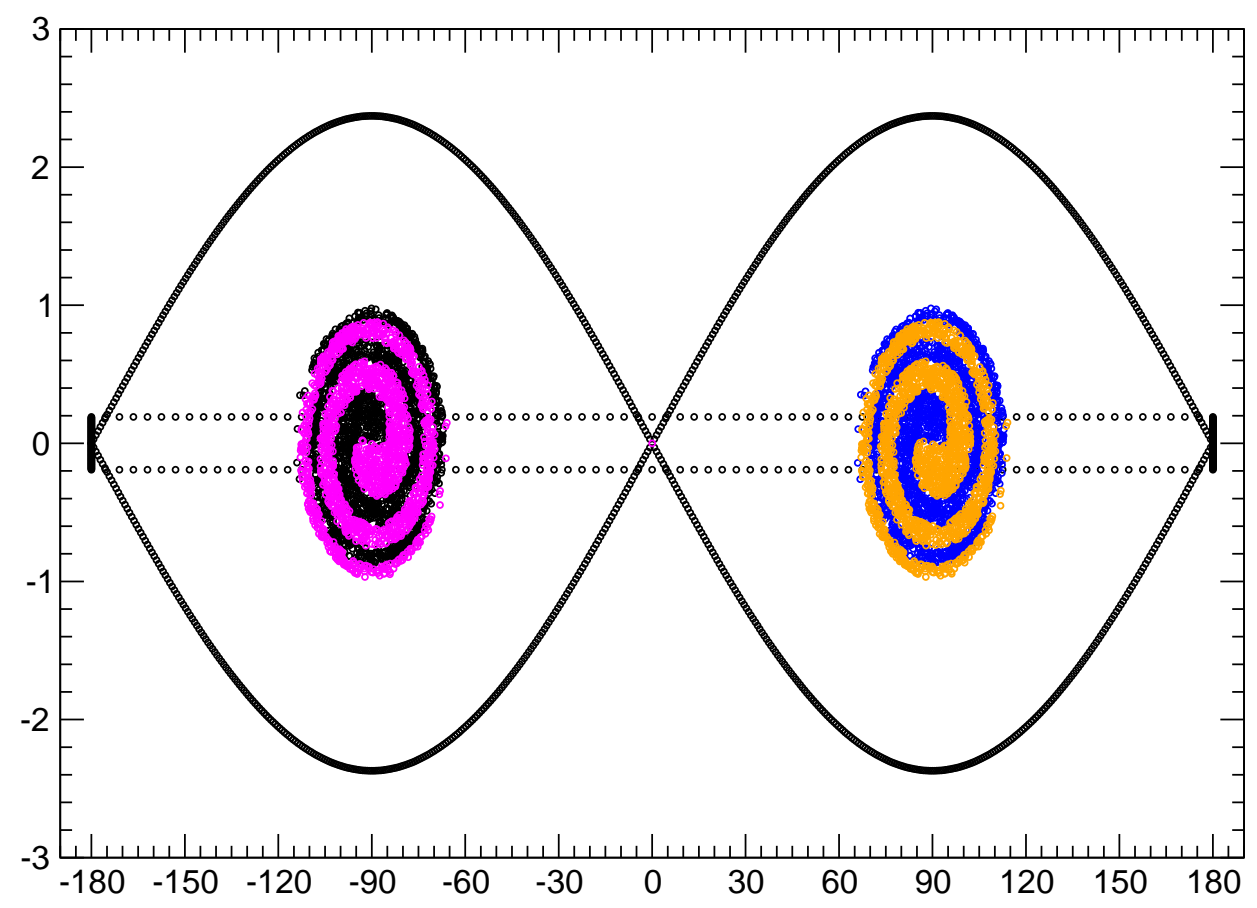

Figure 5: Completion of the 4 to 2 merge. This is at time $80 \mathrm{~ms}$ in Figure 2 and corresponds to the blue curve in Figure 3. The horizontal axis is the phase $\phi$ in degrees. The vertical axis is $W$ in units of eV s. 


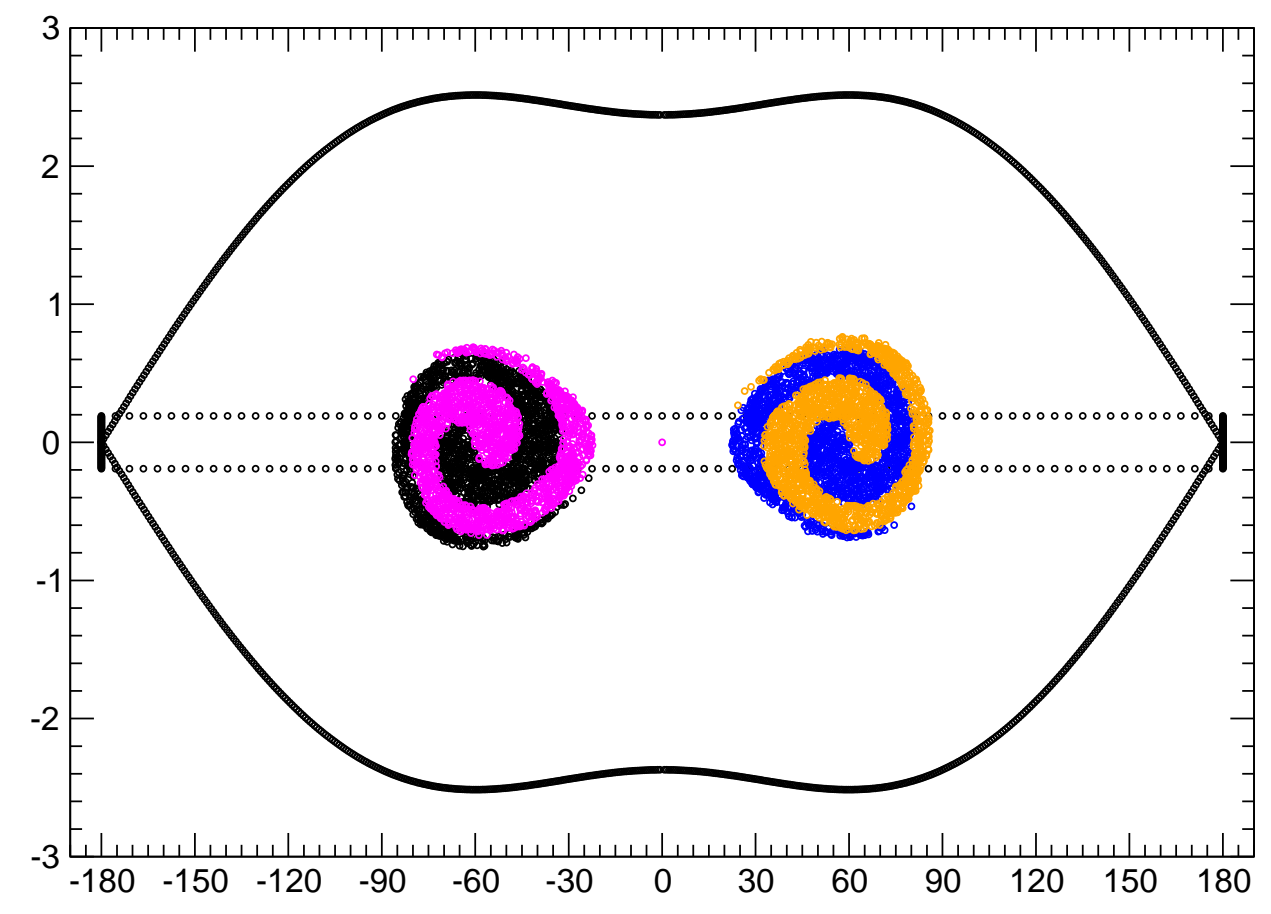

Figure 6: Halfway through the 2 to 1 merge of the bunches in Figure 5 . This is at time $120 \mathrm{~ms}$ in Figure $\mathbf{2}$ and corresponds to the orange curve in Figure 3. The horizontal axis is the phase $\phi$ in degrees. The vertical axis is $W$ in units of eVs. 


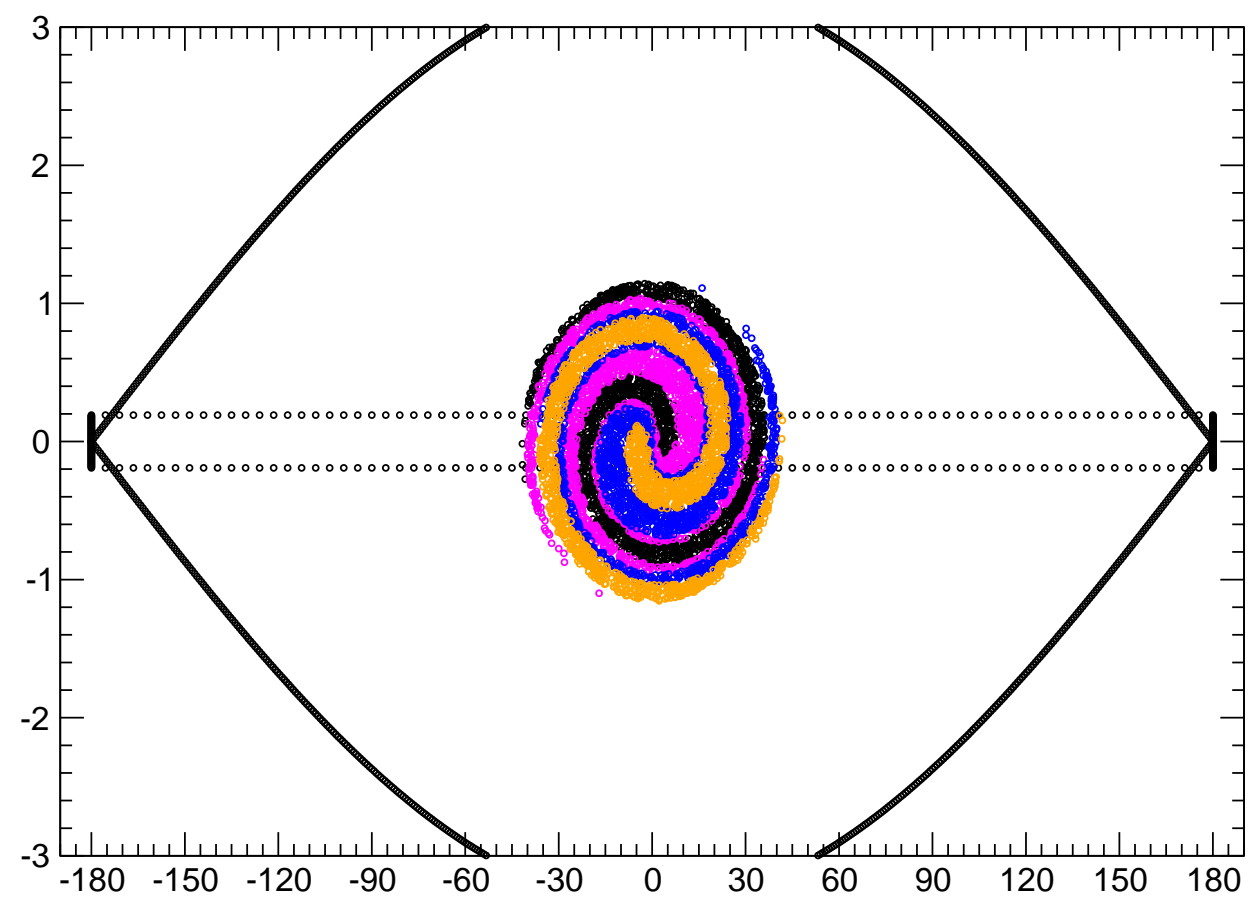

Figure 7: Completion of the 2 to 1 merge. This is at time $160 \mathrm{~ms}$ in Figure 2 and corresponds to the green curve in Figure 3. One can see by inspection (of this and the previous figures) that the emittance of the bunch is close to that $(0.80 \mathrm{eV}$ s per nucleon) of the initial distribution of unbunched beam. The bunch is easily held in the harmonic 2 bucket. The horizontal axis is the phase $\phi$ in degrees. The vertical axis is $W$ in units of eVs. 


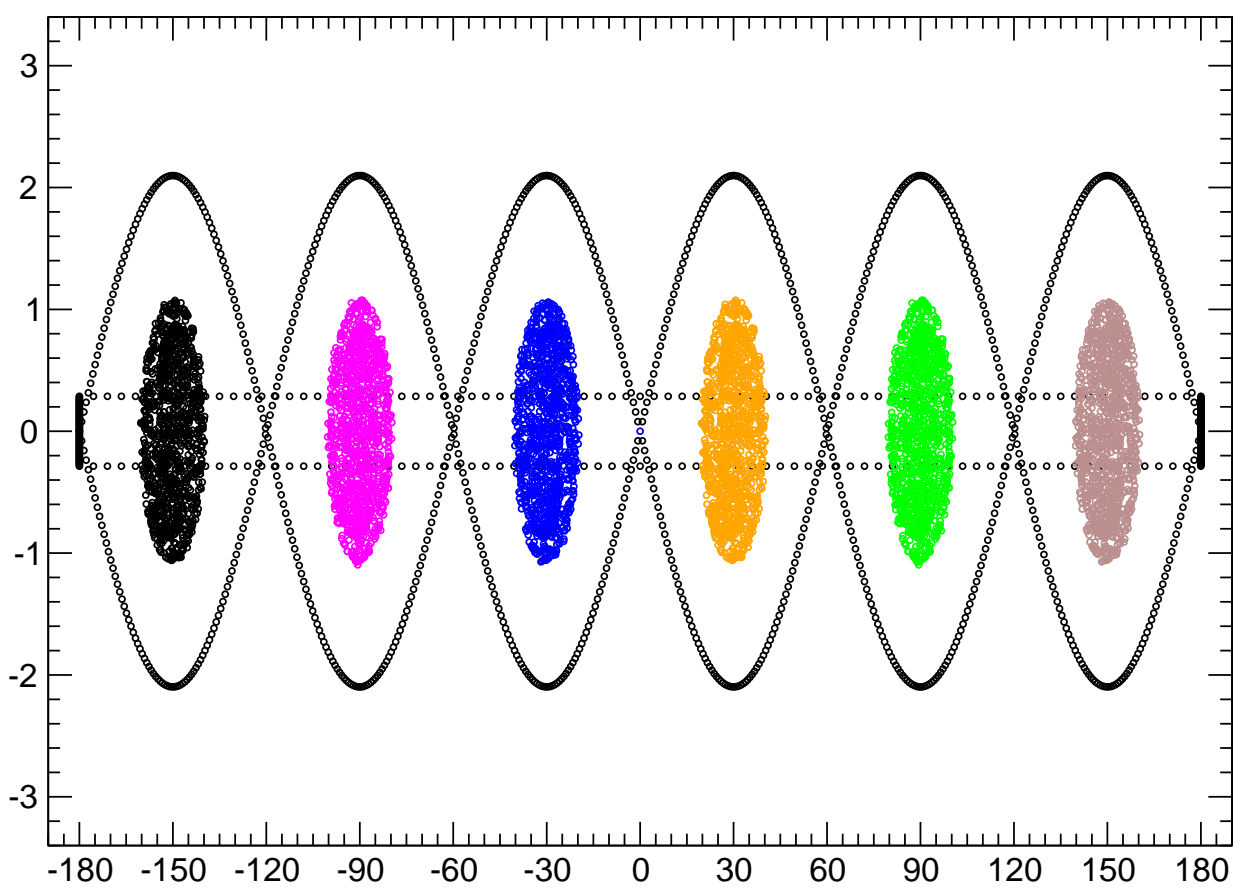

Figure 8: Six bunches of helions in harmonic 12 buckets on the AGS injection porch. The harmonic 12 frequency is $12 f_{s}=3.765 \mathrm{MHz}$. The voltage is $V_{C}=47 \mathrm{kV}$. The black rectangle is the border of the uniform distribution used to make the bunches. The longitudinal emittance of the distribution is $1.20 \mathrm{eV}$ s per nucleon. One can see by inspection that the emittance of the 6 bunches is close to that of the initial distribution. This gives an emittance of $0.2 \mathrm{eV}$ s per nucleon per bunch. In practice the two outer buckets are left unoccupied to provide time for the injection kicker pulse. The horizontal axis is the phase $\phi$ in degrees. The vertical axis is $W$ in units of eVs. 


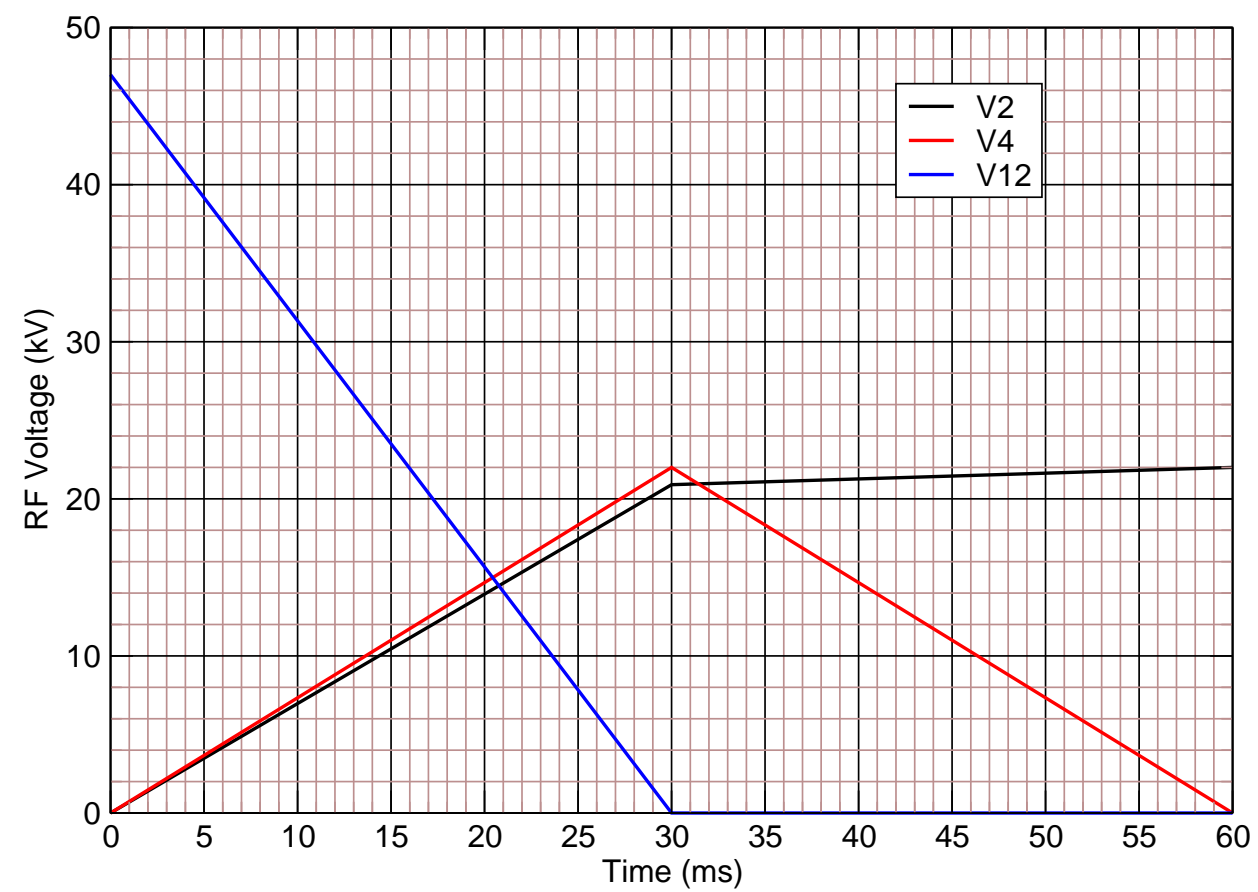

Figure 9: RF voltages for the alternative 4 to 1 merge. Here the black, red, and blue curves are the harmonic $2=1 h, 4=2 h$, and $12=6 h$ voltages respectively. These are given by the formulae of Section 11 with $h$ set equal to 2 . Here the harmonic 12,4 , and 2 voltages are $V_{C}=47 \mathrm{kV}, V_{K}=22$ $\mathrm{kV}$, and $V_{L}=22 \mathrm{kV}$ respectively. The times $T_{K}$ and $T_{L}$ are 30 and 60 ms respectively. The parameter $\mathcal{F}$ has been set equal to 0.95 . This setting keeps the inner 4 bunches separated from the outer 2 during the merging process and minimizes the gross emittance of the final merged bunch. 


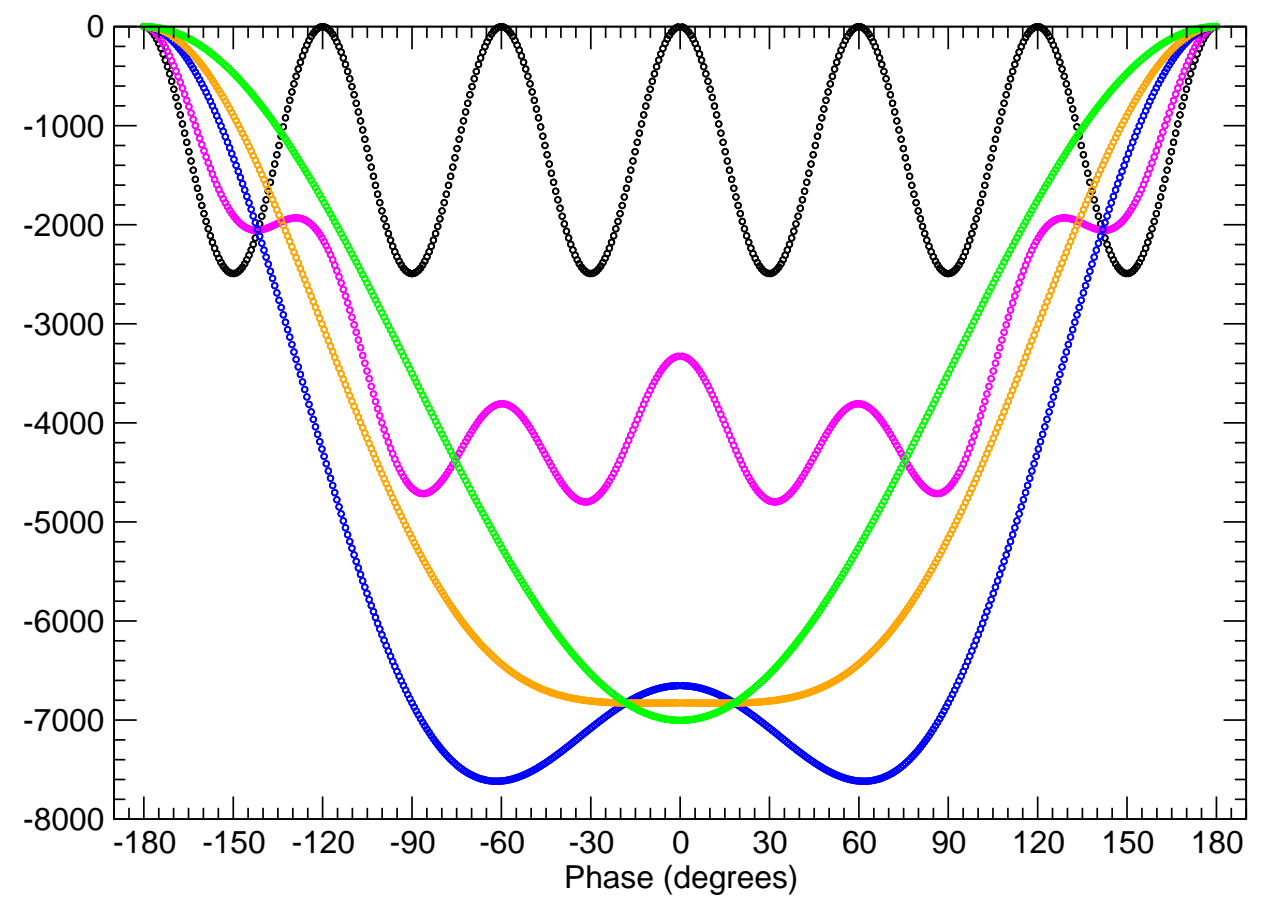

Figure 10: Plots of the "potential" $U\left(\phi_{u}\right)-U(\phi)$ at various times during the alternate 4 to 1 merge. Here $U\left(\phi_{u}\right)$ and $U(\phi)$ are defined in Sections 8 and 11. The black, magenta, blue, orange, and green curves are $U\left(\phi_{u}\right)-U(\phi)$ at times $0,15,30,45$, and $60 \mathrm{~ms}$ respectively. The local minima of the curves correspond to stable fixed points. The local maxima correspond to unstable fixed points. 


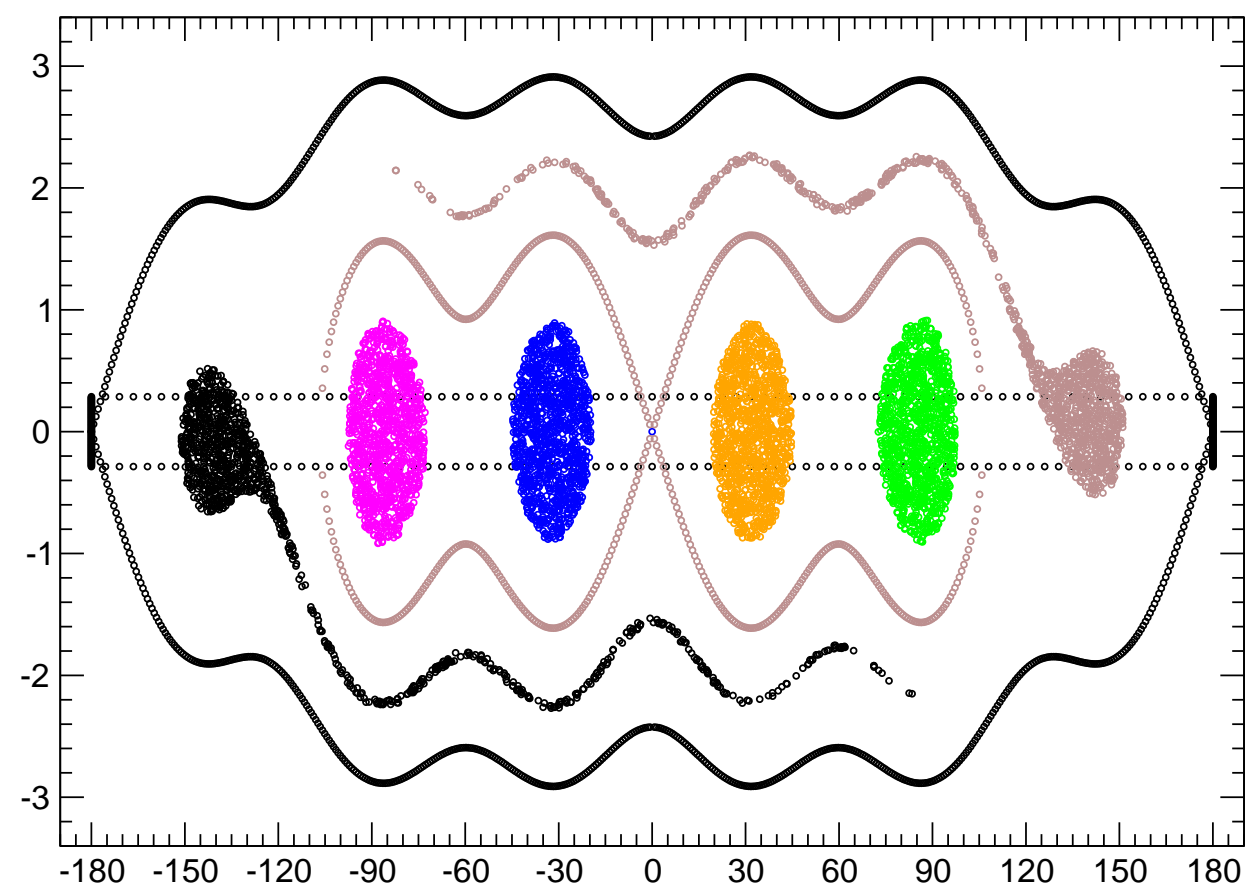

Figure 11: Halfway through the 4 to 2 merge of the bunches in Figure 8. This is at time $15 \mathrm{~ms}$ in Figure $\mathbf{9}$ and corresponds to the magenta curve in Figure 10. Note that the brown separatrix keeps the inner 4 bunches separated from the outer 2. The two outer bunches (which are not present in practice) have started to form a halo around the inner 4 . The horizontal axis is the phase $\phi$ in degrees. The vertical axis is $W$ in units of eVs. 


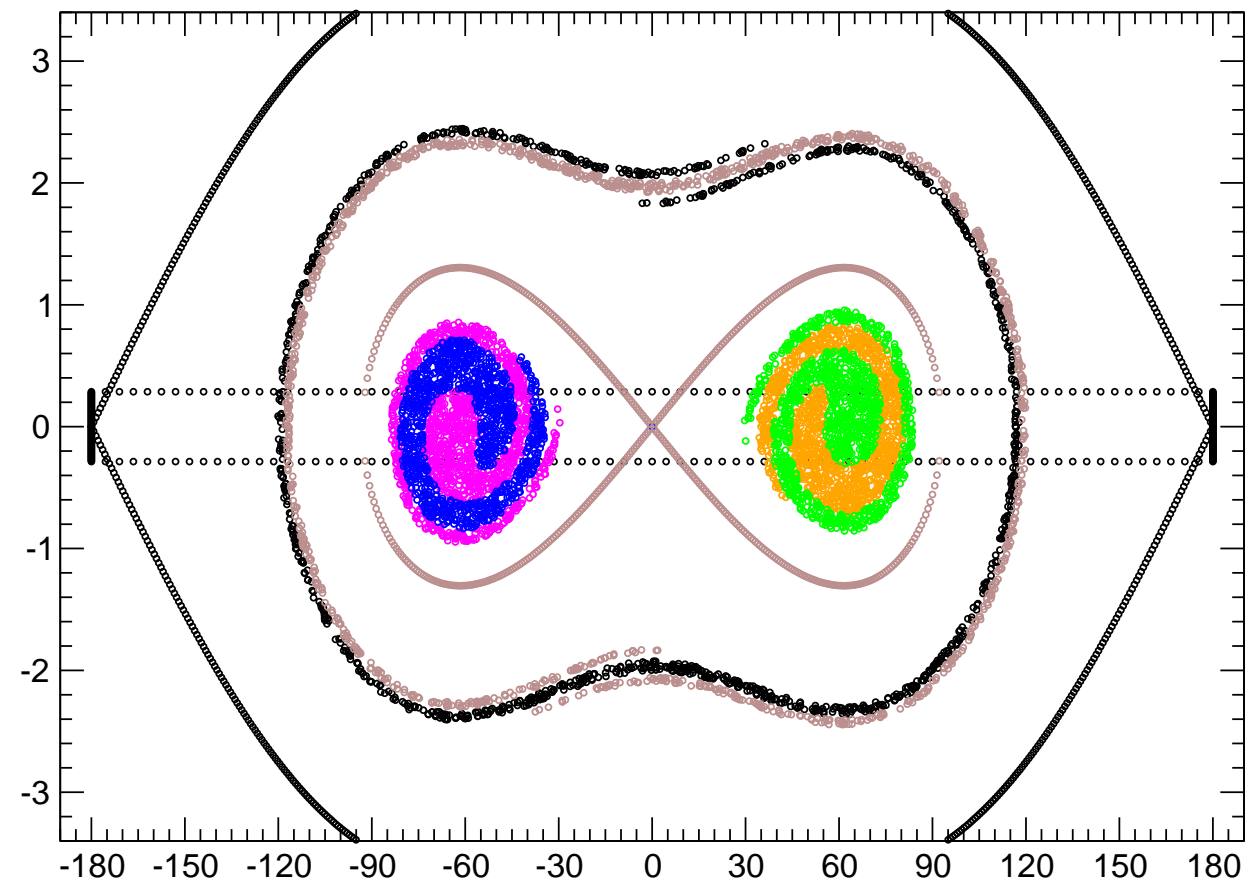

Figure 12: Completion of the 4 to 2 merge. This is at time $30 \mathrm{~ms}$ in Figure 9 and corresponds to the blue curve in Figure 10. Note again that the brown separatrix keeps the inner 4 bunches separated from the outer 2. The two outer bunches (which are not present in practice) have formed a halo around the inner 4 . The horizontal axis is the phase $\phi$ in degrees. The vertical axis is $W$ in units of eV s. 


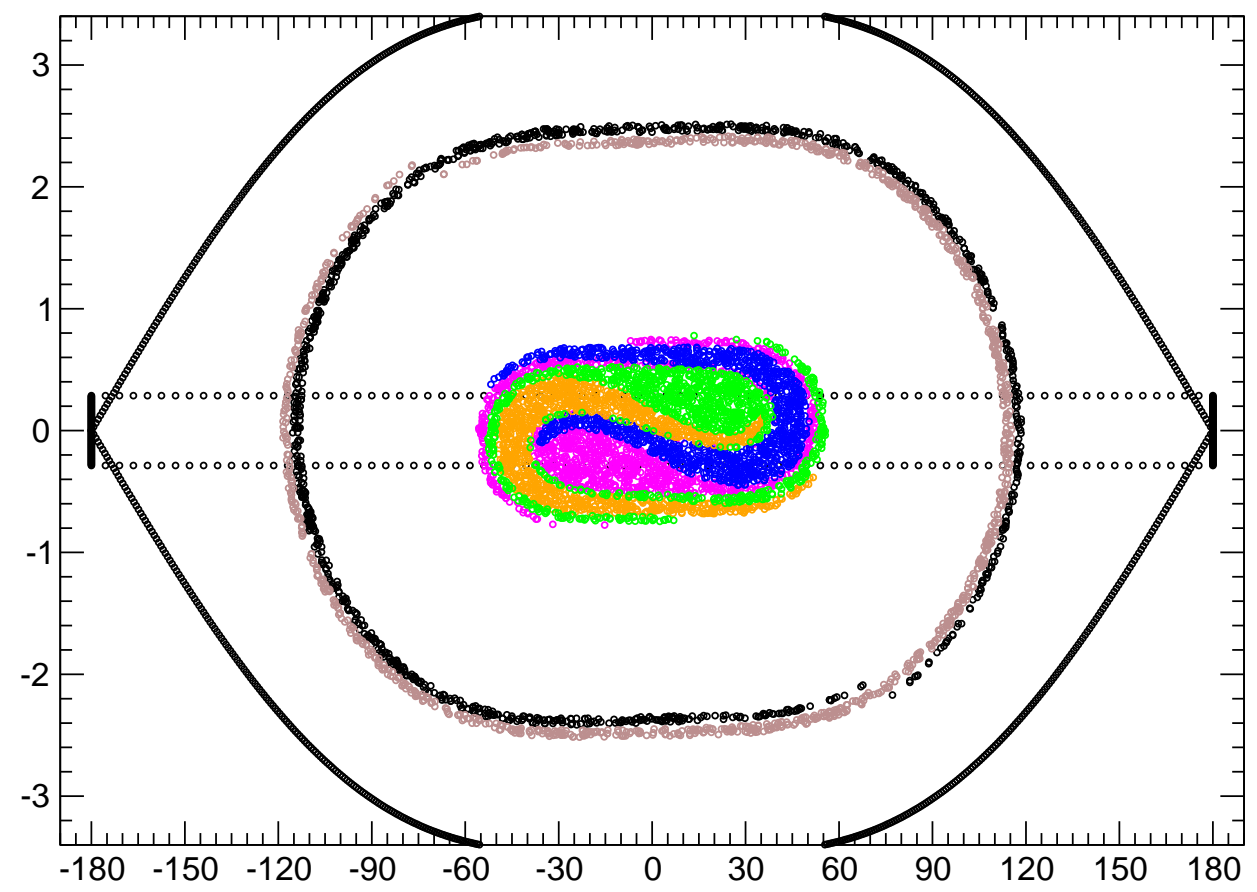

Figure 13: Halfway through the 2 to 1 merge of the bunches in Figure 12. This is at time $45 \mathrm{~ms}$ in Figure $\mathbf{9}$ and corresponds to the orange curve in Figure 10. The horizontal axis is the phase $\phi$ in degrees. The vertical axis is $W$ in units of eVs. 


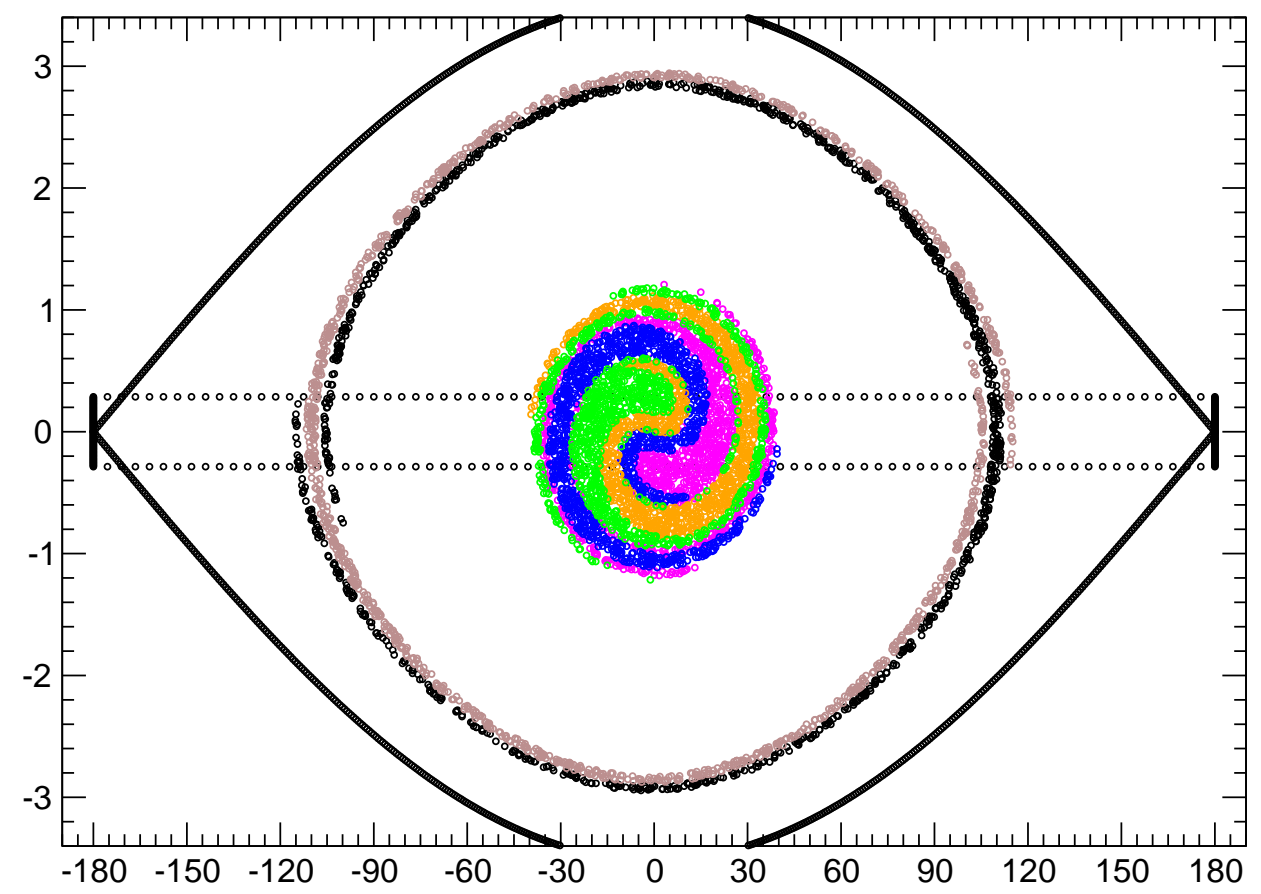

Figure 14: Completion of the 2 to 1 merge. This is at time $60 \mathrm{~ms}$ in Figure 9 and corresponds to the green curve in Figure 10. One can see by inspection (of this and the previous figures) that the emittance of the merged bunch is close to that $(0.80 \mathrm{eV} \mathrm{s}$ per nucleon) of the 4 inner bunches of the initial distribution of bunched beam. The bunch is easily held in the harmonic 2 bucket. (The halo, formed by the 2 outer bunches of the initial distribution of bunched beam, is not present in practice.) The horizontal axis is the phase $\phi$ in degrees. The vertical axis is $W$ in units of eVs. Comparing with Figure 7 one sees that alternative 4 to 1 merge produces a merged bunch of the same size and quality as the standard 4 to 1 merge. 


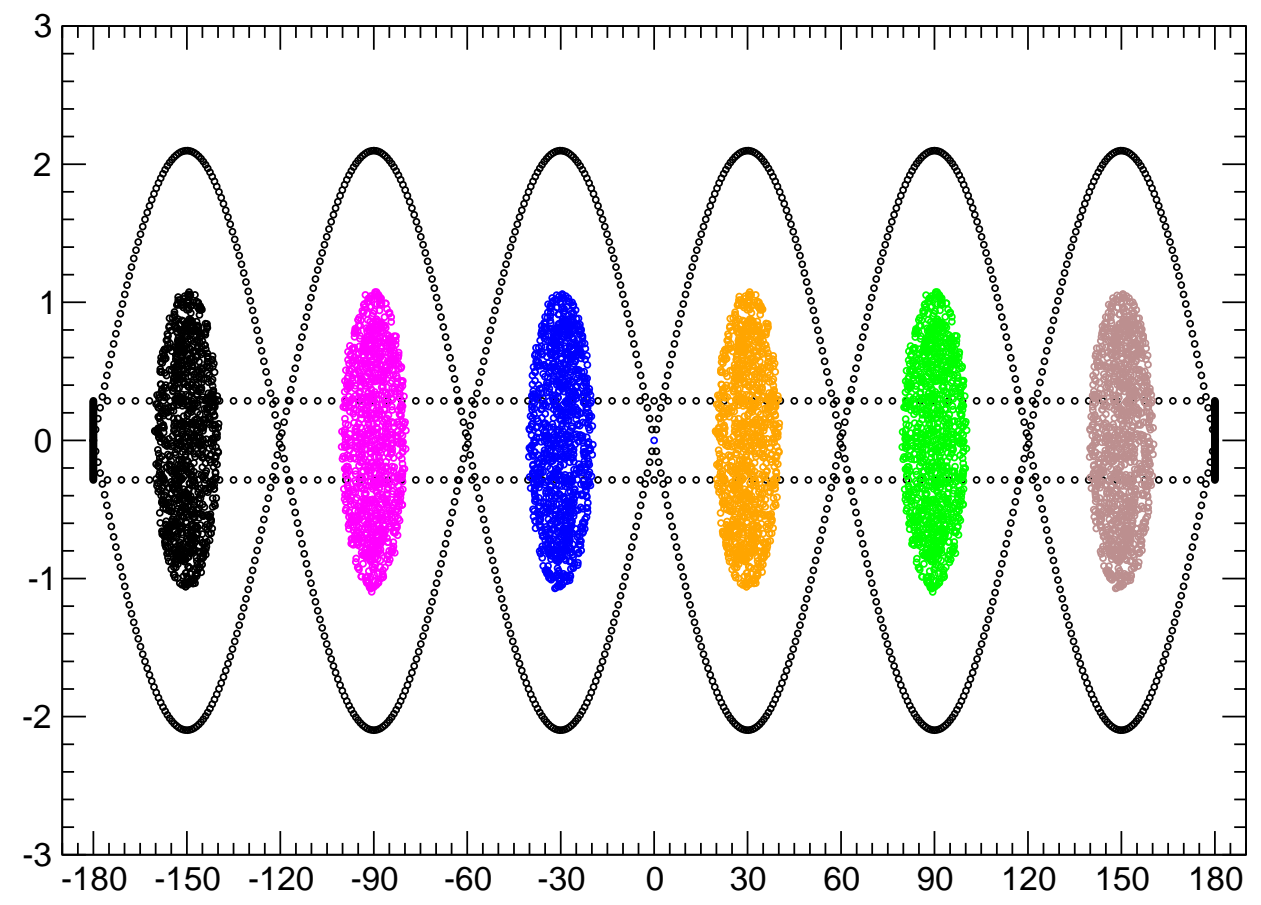

Figure 15: Six bunches of helions in harmonic 12 buckets on the AGS injection porch. The harmonic 12 frequency is $12 f_{s}=3.765 \mathrm{MHz}$. The RF voltage is $V_{C}=47 \mathrm{kV}$. The black rectangle is the border of the uniform distribution used to make the bunches. The longitudinal emittance of the distribution is $1.20 \mathrm{eV}$ s per nucleon. One can see by inspection that the emittance of the 6 bunches is close to that of the initial distribution. This gives an emittance of $0.2 \mathrm{eV}$ s per nucleon per bunch. The horizontal axis is the phase $\phi$ in degrees. The vertical axis is $W$ in units of eVs. 


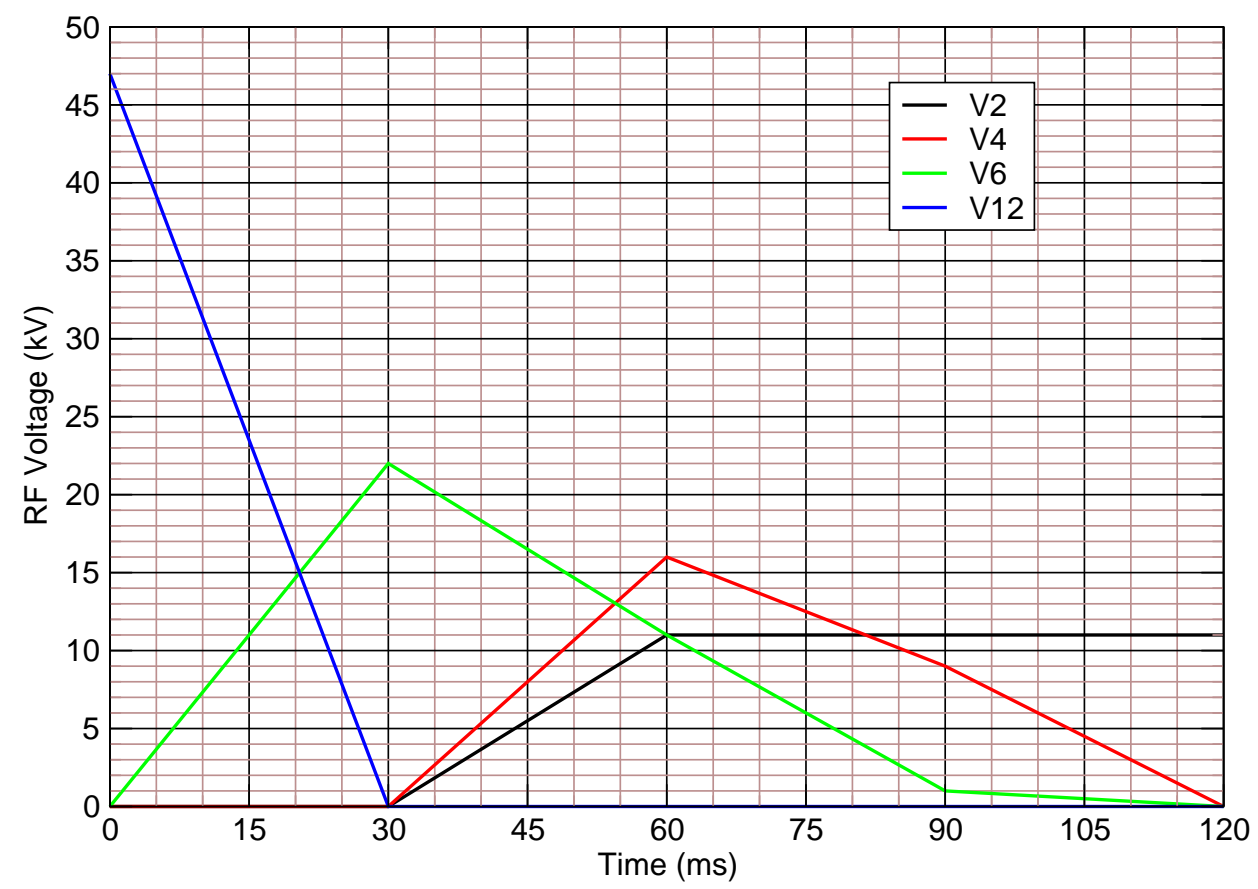

Figure 16: RF voltages for the 6 to 1 merge. Here the black, red, green, and blue curves are the harmonic $2=1 h, 4=2 h, 6=3 h$, and $12=6 h$ voltages respectively. These are given by the formulae of Section 12 with $h$ set equal to 2 . Here the harmonic 12 voltage $V_{C}=47 \mathrm{kV}$. The harmonic 6 voltages $V_{M}=22 \mathrm{kV}, V_{M 1}=11 \mathrm{kV}$, and $V_{M 2}=1.0 \mathrm{kV}$. The harmonic 4 voltages $V_{K 1}=16 \mathrm{kV}$ and $V_{K 2}=9 \mathrm{kV}$. The harmonic 2 voltage $V_{L 1}=11 \mathrm{kV}$. The times $T_{K}, T_{L 1}, T_{L 2}$, and $T_{F}$ are $30,60,90$, and $120 \mathrm{~ms}$ respectively. The 6 to 3 merge takes place over the time interval $0 \leq T \leq 30 \mathrm{~ms}$. The 3 to 1 merge takes place over the interval $30 \leq T \leq 120 \mathrm{~ms}$. 


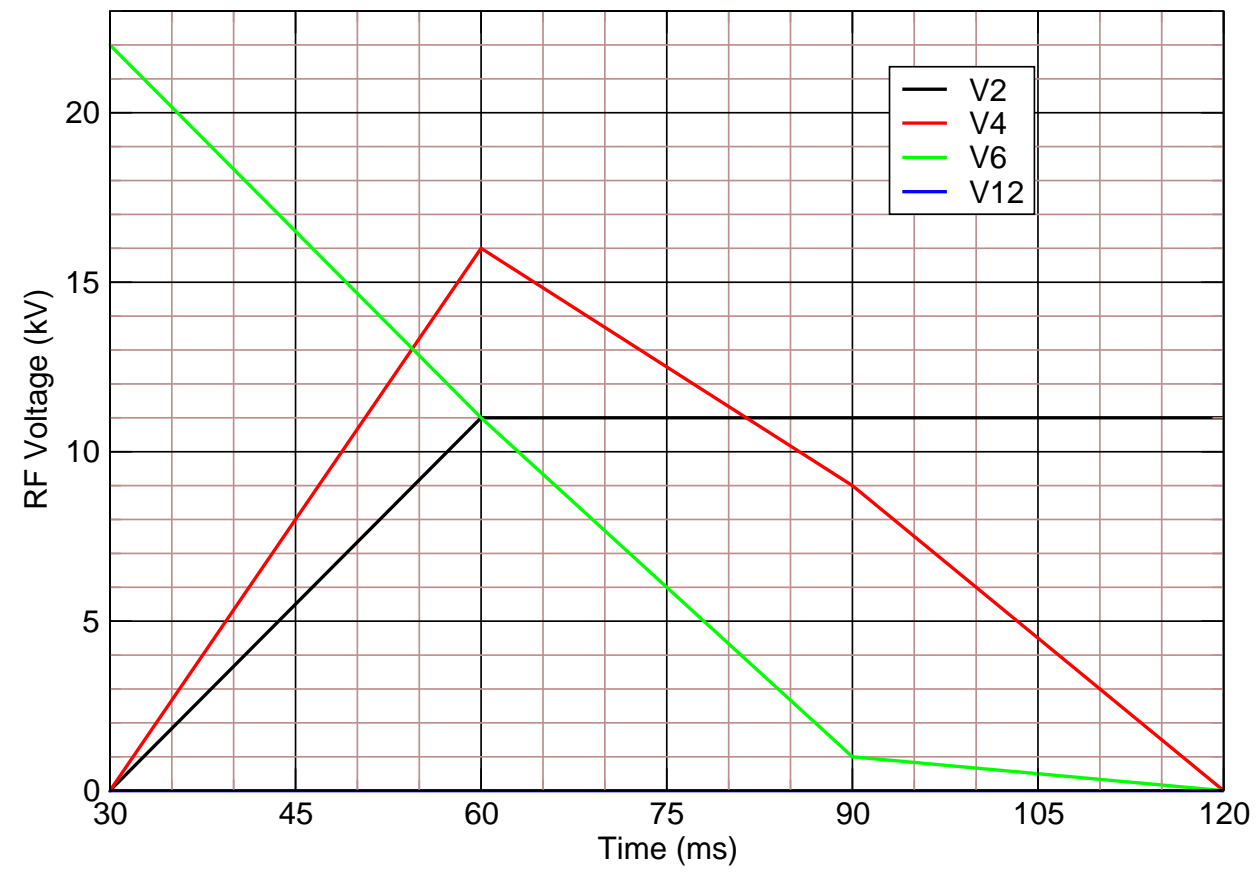

Figure 17: Magnified view of Figure 16 showing the RF voltages for the 3 to 1 portion of the 6 to 1 merge. 


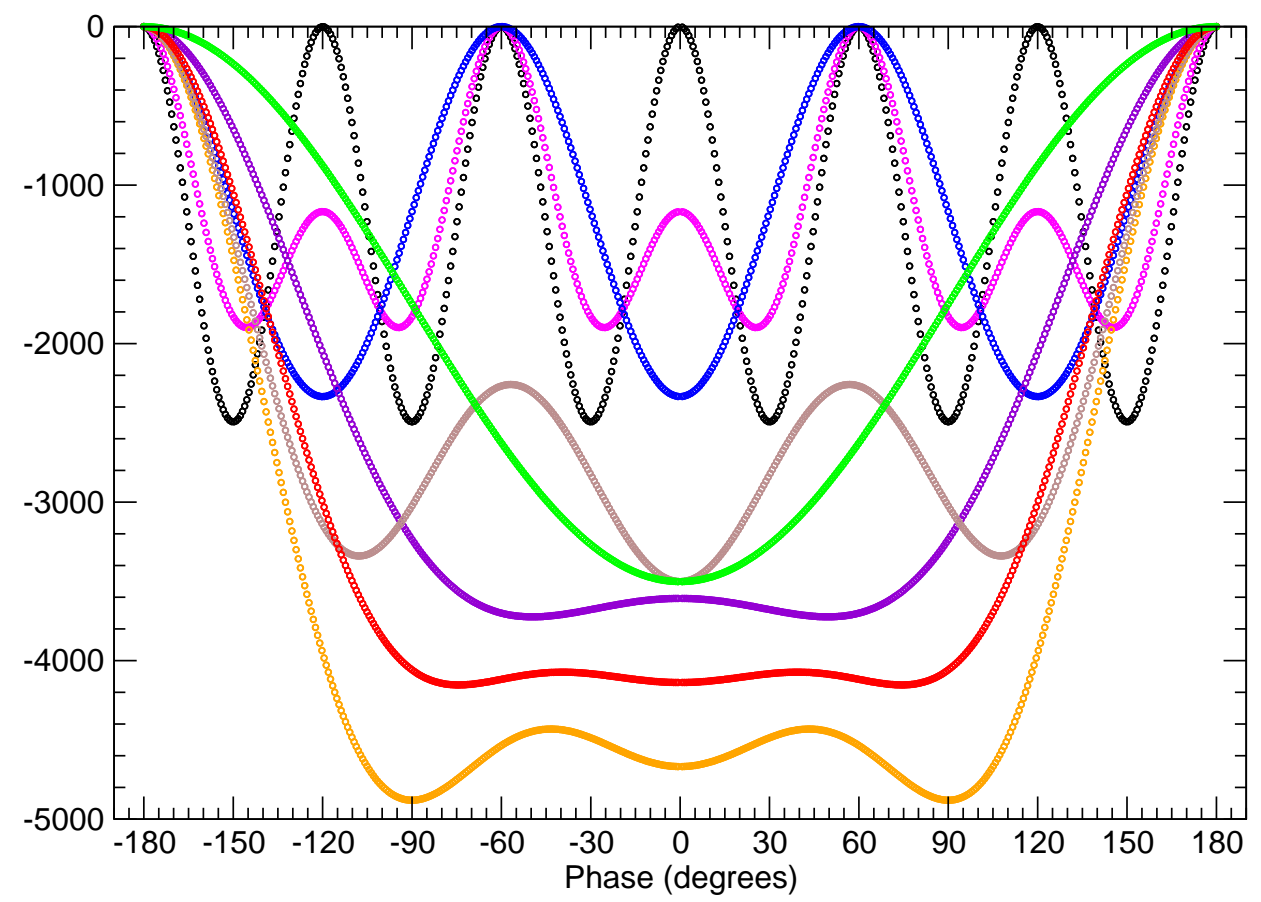

Figure 18: Plots of the "potential" $U\left(\phi_{u}\right)-U(\phi)$ at various times during the 6 to 1 merge. Here $U\left(\phi_{u}\right)$ and $U(\phi)$ are defined in Sections 8 and 12 . The black, magenta, blue, brown, orange, red, violet, and green curves are $U\left(\phi_{u}\right)-U(\phi)$ at times $0,15,30,45,60,75,90$, and $120 \mathrm{~ms}$ respectively. The local minima of the curves correspond to stable fixed points. The local maxima correspond to unstable fixed points. 


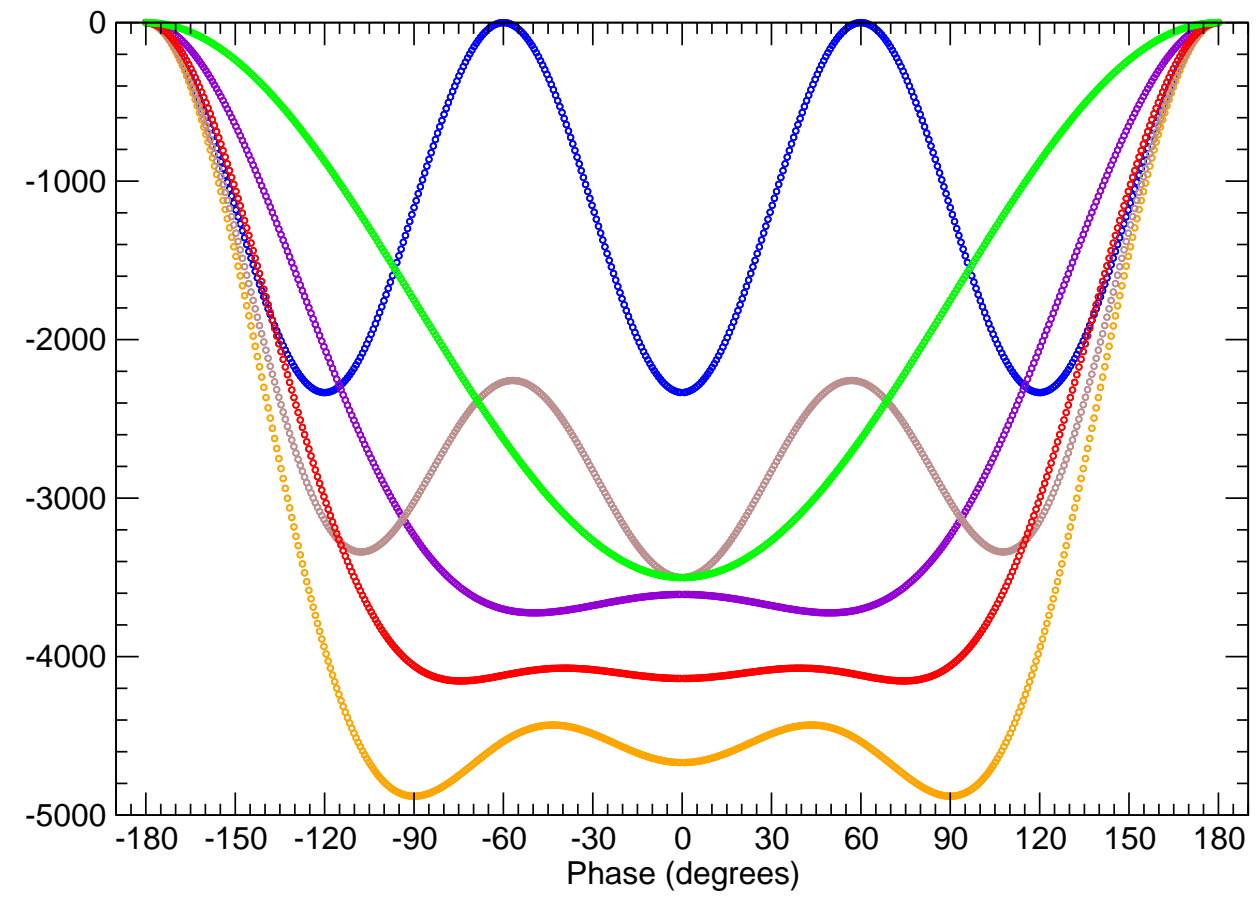

Figure 19: Plots of the "potential" $U\left(\phi_{u}\right)-U(\phi)$ at various times during the 3 to 1 portion of 6 to 1 merge. Here $U\left(\phi_{u}\right)$ and $U(\phi)$ are defined in Sections 8 and 12. The blue, brown, orange, red, violet, and green curves are $U\left(\phi_{u}\right)-U(\phi)$ at times $30,45,60,75,90$, and $120 \mathrm{~ms}$ respectively. The local minima of the curves correspond to stable fixed points. The local maxima correspond to unstable fixed points. 


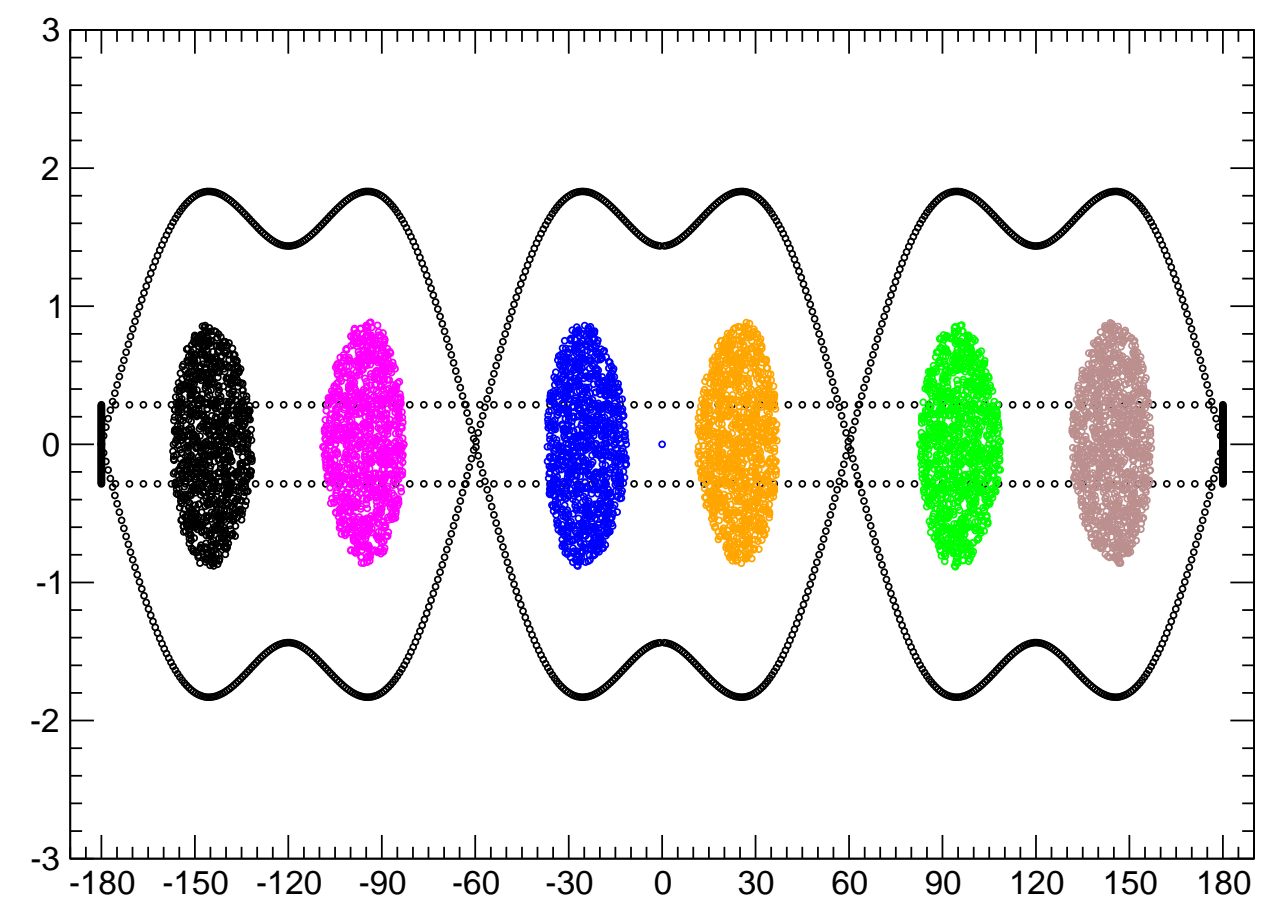

Figure 20: Halfway through the 6 to 3 merge of the bunches in Figure 15. This is at time $15 \mathrm{~ms}$ in Figure $\mathbf{1 6}$ and corresponds to the magenta curve in Figure 18. The horizontal axis is the phase $\phi$ in degrees. The vertical axis is $W$ in units of eVs. 


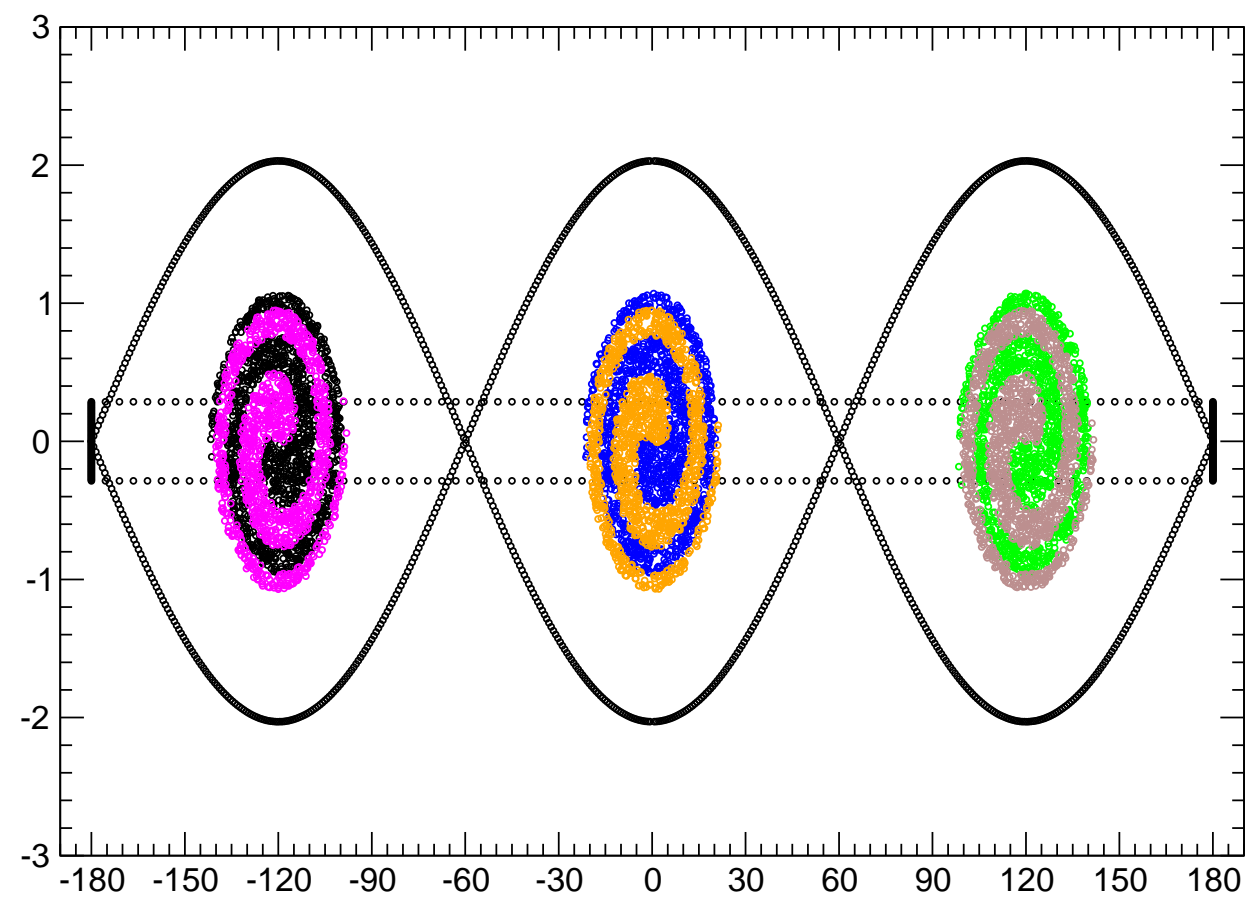

Figure 21: Completion of the 6 to 3 merge. This is at time $30 \mathrm{~ms}$ in Figure 16 and corresponds to the blue curve in Figure 18. The horizontal axis is the phase $\phi$ in degrees. The vertical axis is $W$ in units of eVs. 


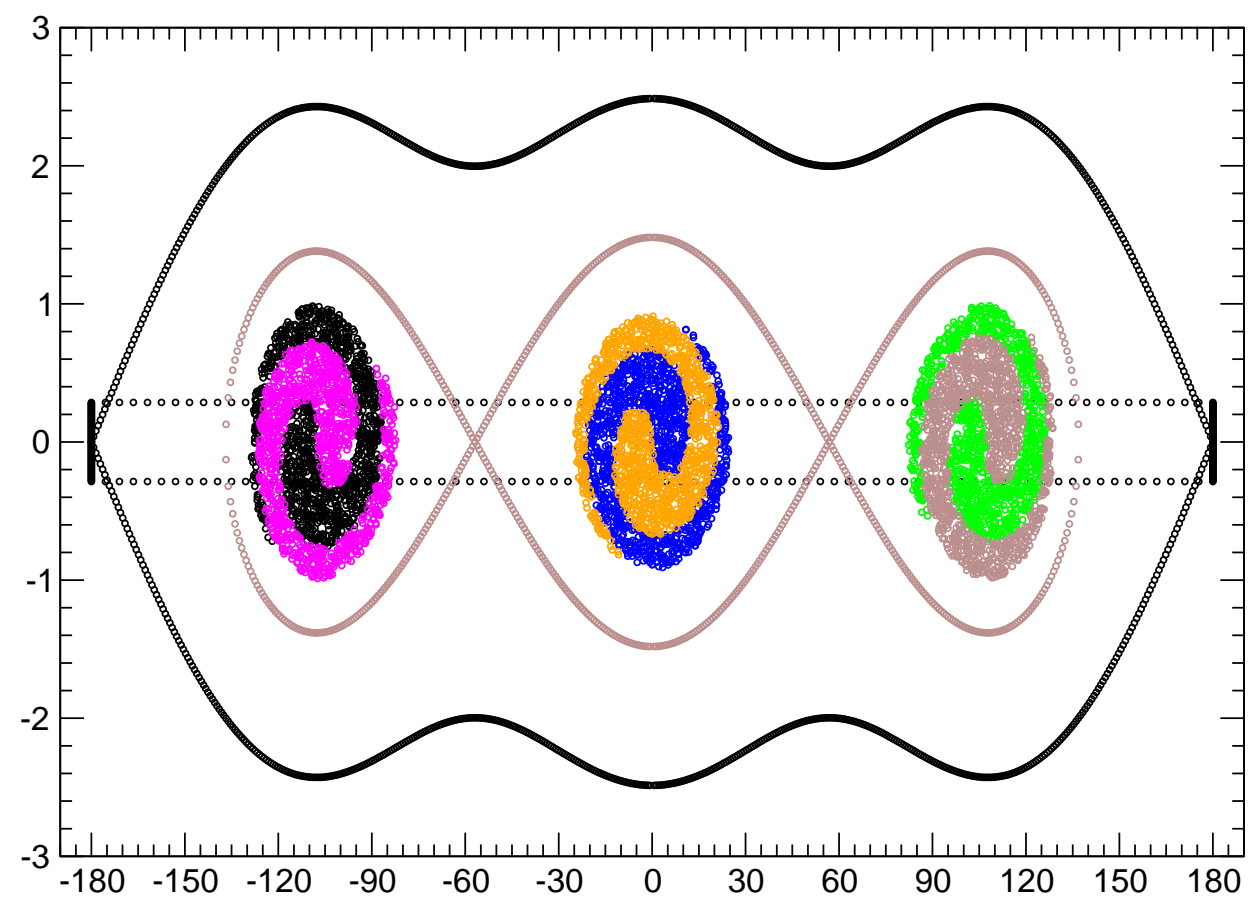

Figure 22: One sixth of the way through the 3 to 1 merge of the bunches in Figure 21. This is at time $45 \mathrm{~ms}$ in Figure 16 and corresponds to the brown curve in Figure 18. The bunches are kept separated by the brown separatrix. The horizontal axis is the phase $\phi$ in degrees. The vertical axis is $W$ in units of eVs. 


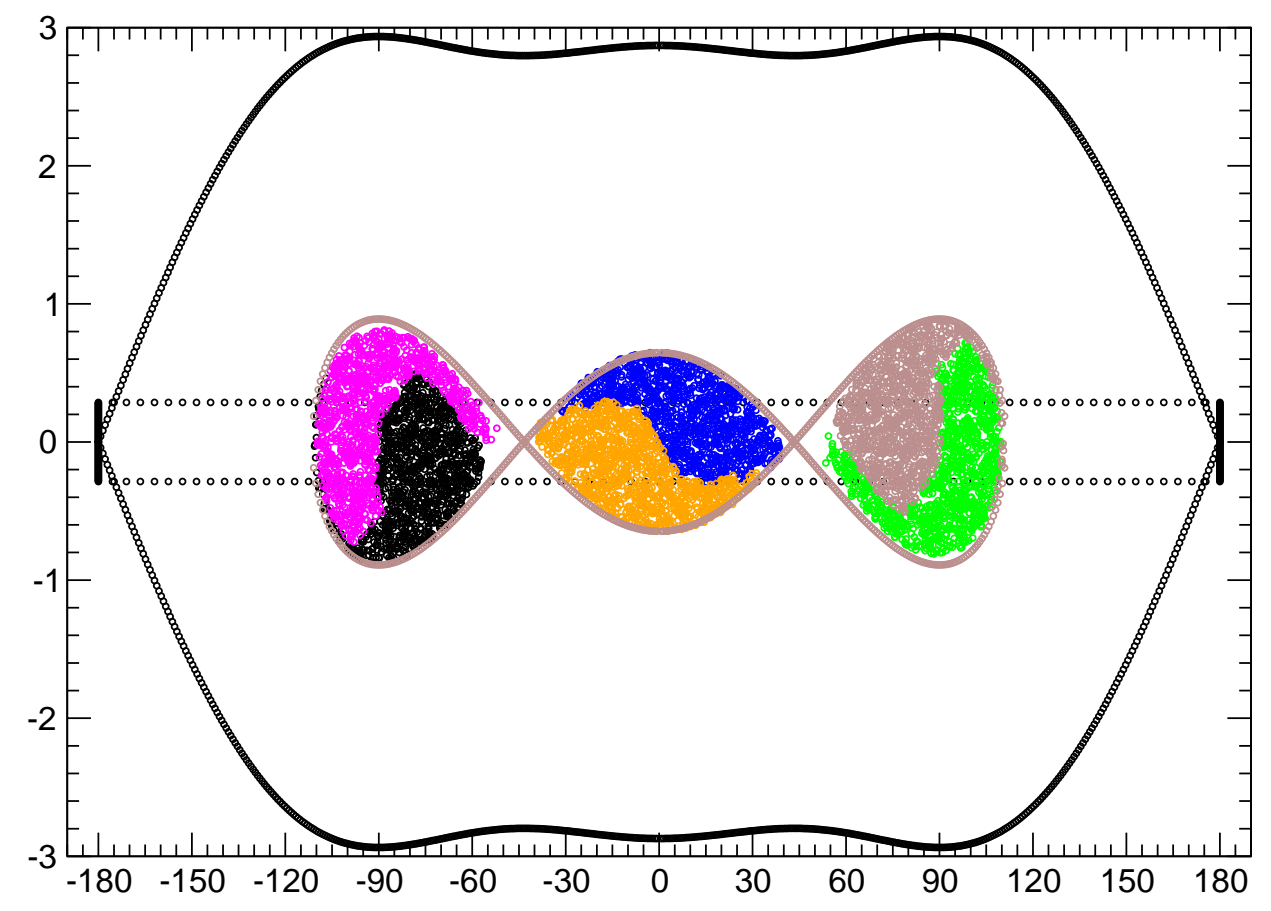

Figure 23: One third of the way through the 3 to 1 merge of the bunches in Figure 21. This is at time $60 \mathrm{~ms}$ in Figure $\mathbf{1 6}$ and corresponds to the orange curve in Figure 18. The bunches are nearly touching but are still in separate buckets. The horizontal axis is the phase $\phi$ in degrees. The vertical axis is $W$ in units of eVs. 


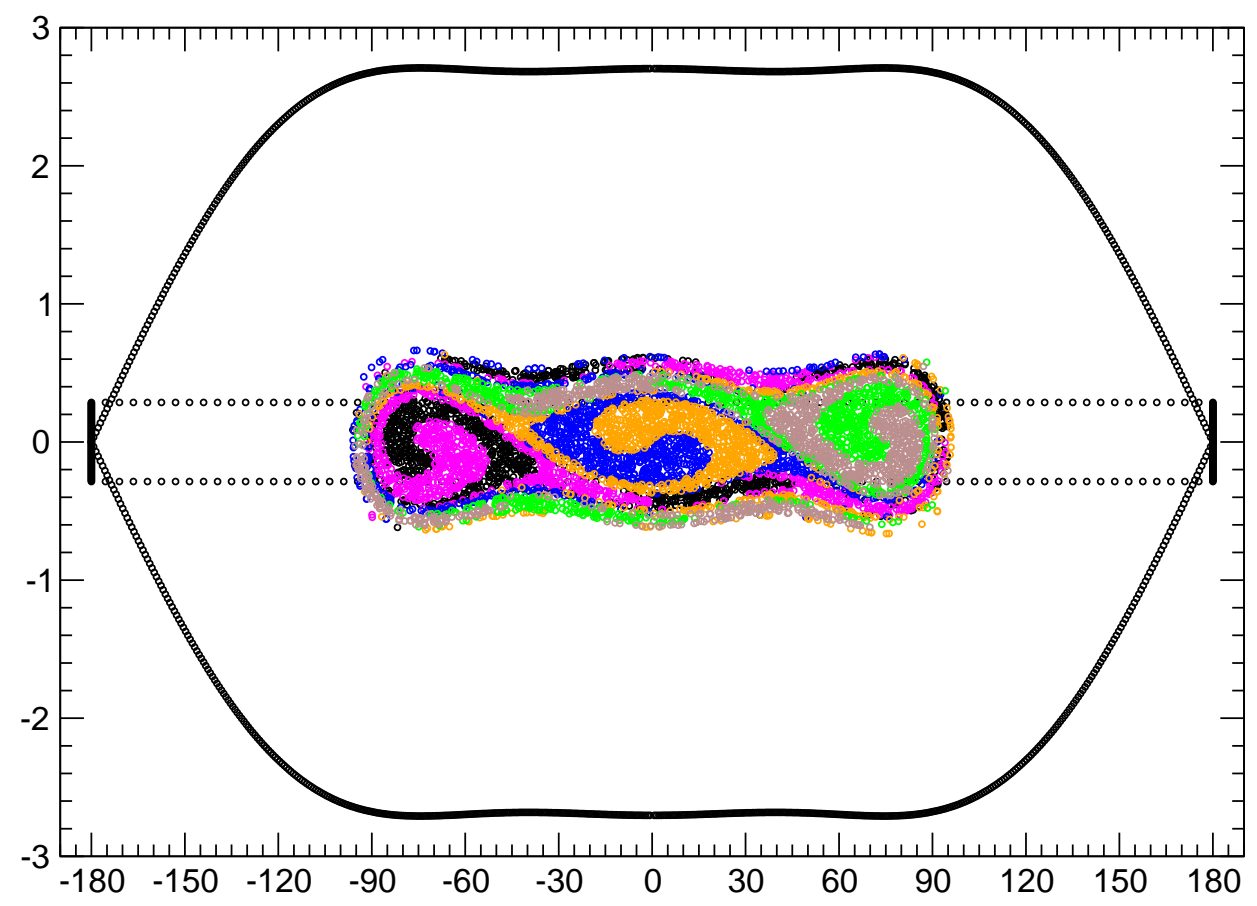

Figure 24: Halfway through the 3 to 1 merge of the bunches in Figure 21. This is at time $75 \mathrm{~ms}$ in Figure $\mathbf{1 6}$ and corresponds to the red curve in Figure 18. Here the bunches have started to mix with one another. The horizontal axis is the phase $\phi$ in degrees. The vertical axis is $W$ in units of eVs. 


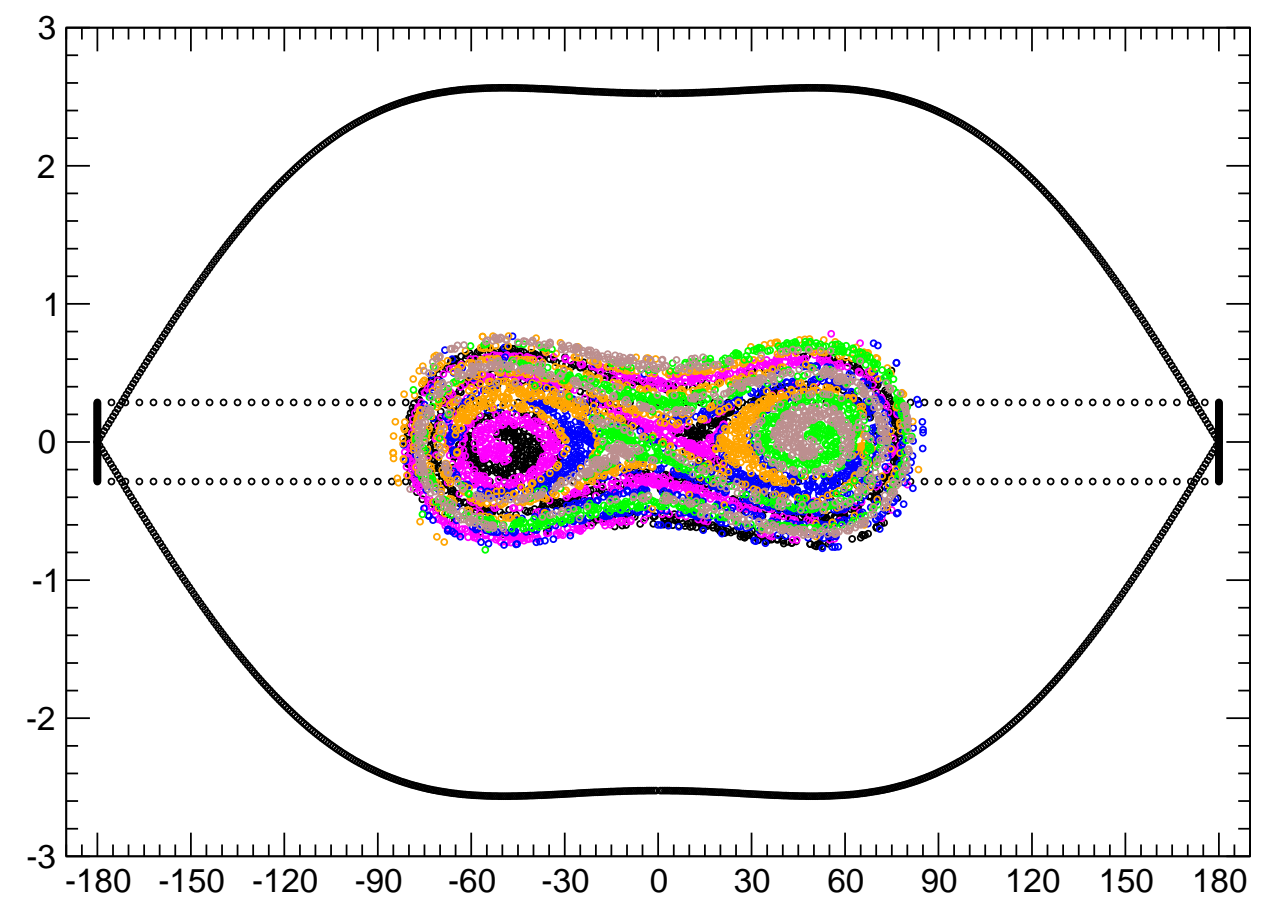

Figure 25: Two thirds of the way through the 3 to 1 merge of the bunches in Figure 21. This is at time $90 \mathrm{~ms}$ in Figure $\mathbf{1 6}$ and corresponds to the violet curve in Figure 18. The bunches continue to mix and merge. The horizontal axis is the phase $\phi$ in degrees. The vertical axis is $W$ in units of eVs. 


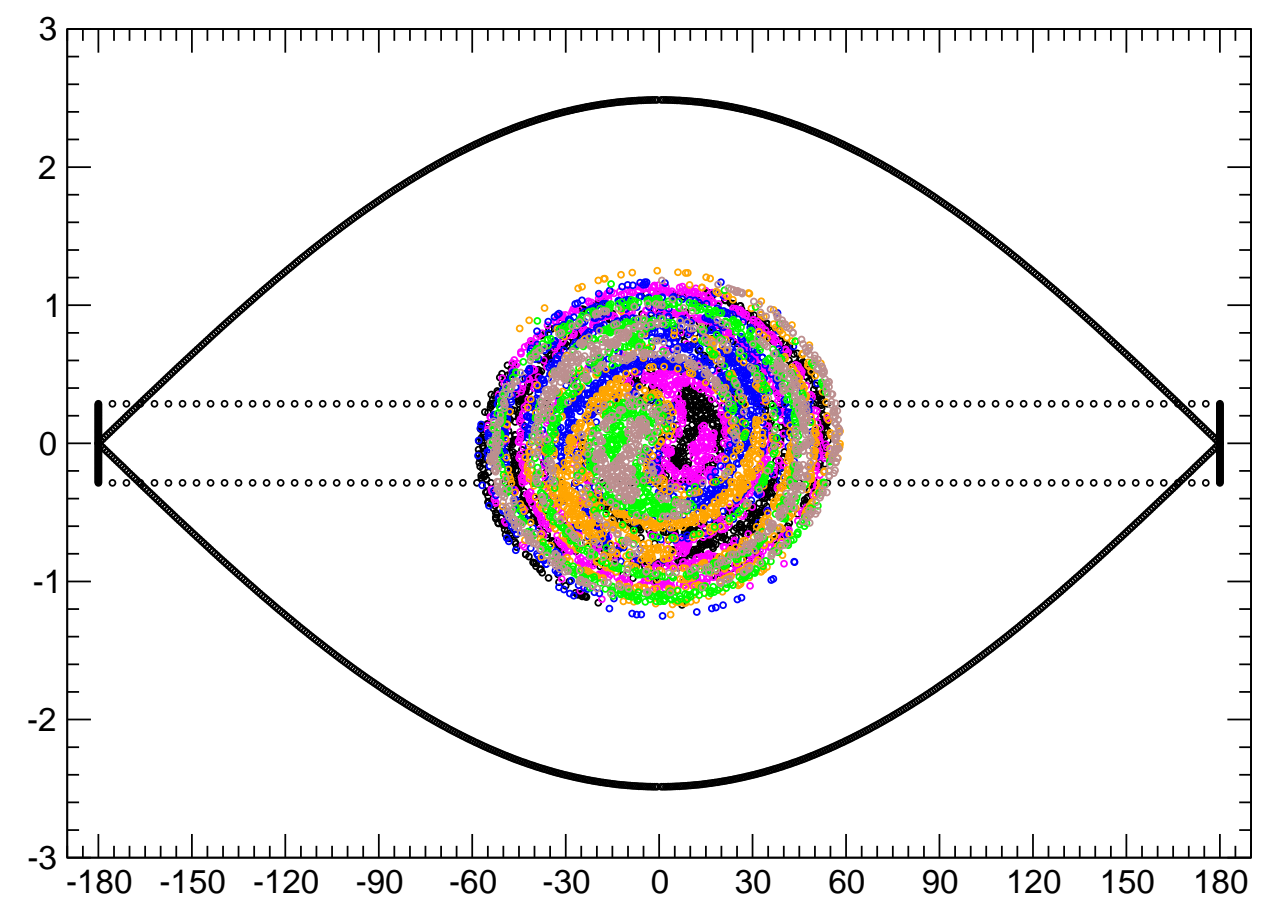

Figure 26: Completion of the 3 to 1 merge of the bunches in Figure 21. This is at time $120 \mathrm{~ms}$ in Figure $\mathbf{1 6}$ and corresponds to the green curve in Figure 18. The horizontal axis is the phase $\phi$ in degrees. The vertical axis is $W$ in units of eVs. 


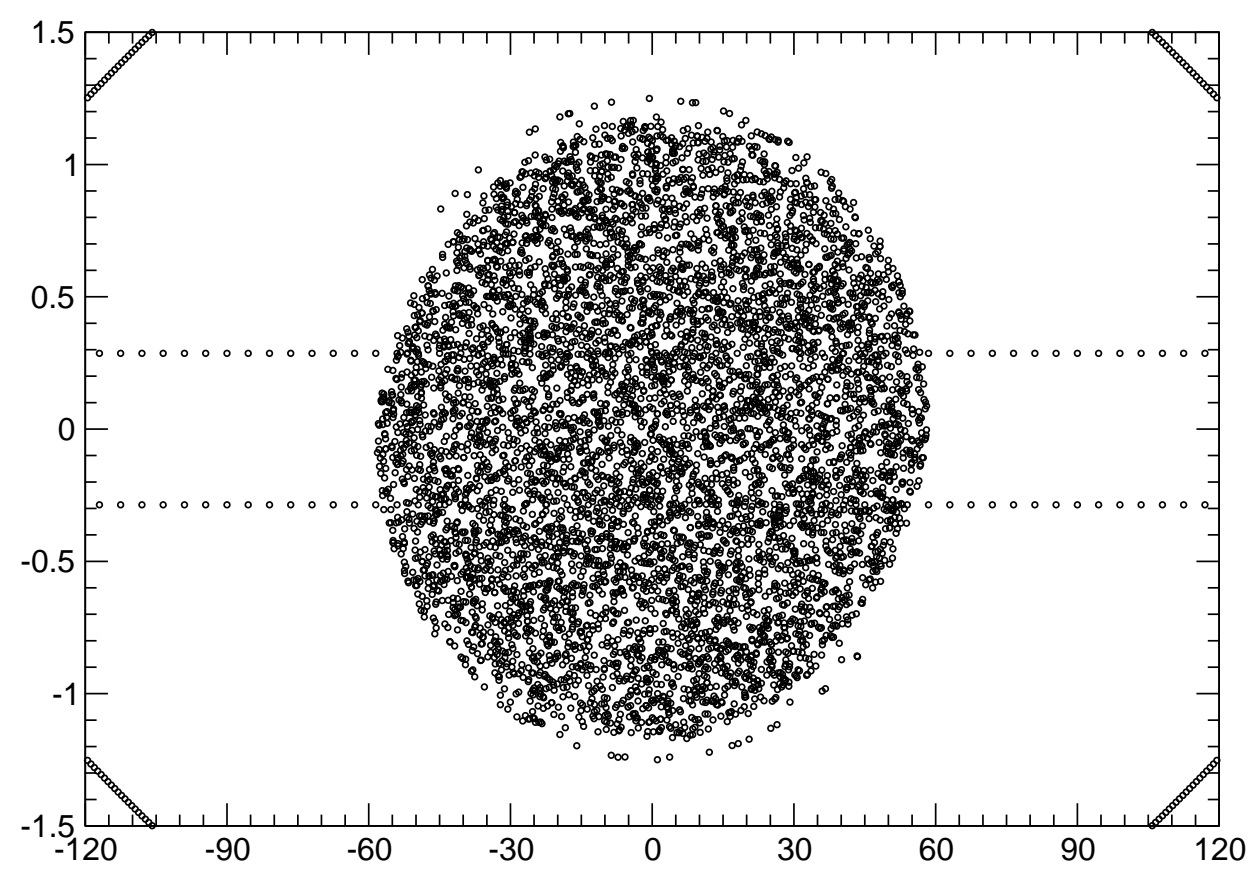

Figure 27: Magnified view of Figure 26 with color suppressed, showing a tightly merged bunch. This is the final result of the 6 to 1 merge. 


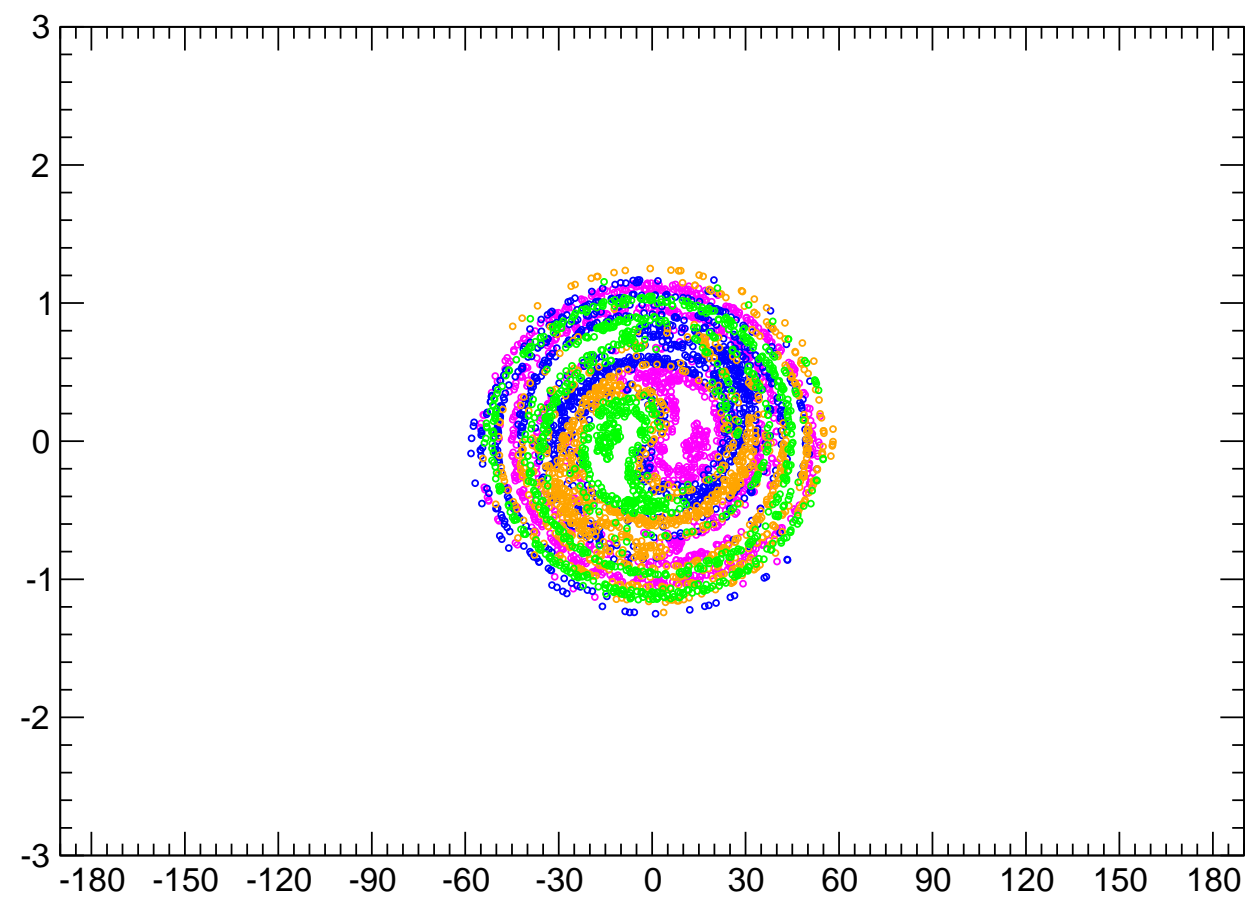

Figure 28: If the 2 outer buckets in Figure 15 are left empty, then the 6 to 1 merge becomes another alternative scheme for merging 4 bunches into 1. In this case, however, the phase space of the 2 outer buckets mixes with the 4 inner bunches and dilutes the gross emittance. One ends up with the bunch shown above. (This was obtained by simply turning off the black and brown points in Figure 26.) The gross emittance of the bunch is $6 / 4$ times larger than that obtained with the alternative 4 to 1 scheme of Section 14. 


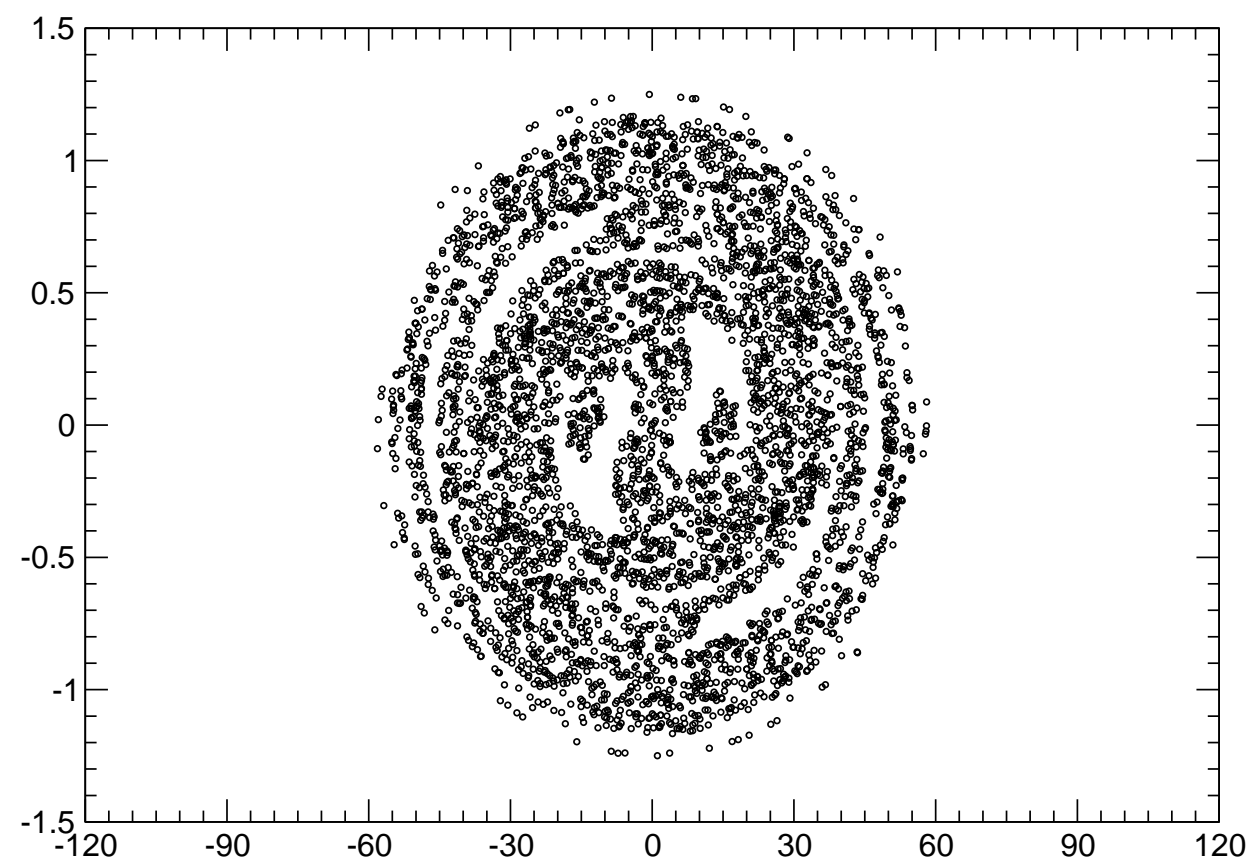

Figure 29: Magnified view of Figure 28 with color suppressed, showing the dilution of the gross emittance. 